\title{
Evidence synthesis in pulmonary arterial hypertension: a systematic review and critical appraisal
}

\author{
Max Schlueter ${ }^{1 *} \mathbb{D}$, Amélie Beaudet ${ }^{2}$, Evan Davies², Binu Gurung ${ }^{1}$ and Andreas Karabis ${ }^{3,4}$
}

\begin{abstract}
Background: The clinical landscape of pulmonary arterial hypertension (PAH) has evolved in terms of disease definition and classification, trial designs, available therapies and treatment strategies as well as clinical guidelines. This study critically appraises published evidence synthesis studies, i.e. meta-analyses (MA) and network-metaanalyses (NMA), to better understand their quality, validity and discuss the impact of the findings from these studies on current decision-making in PAH.

Methods: A systematic literature review to identify MA/NMA studies considering approved and available therapies for treatment of PAH was conducted. Embase, Medline and the Cochrane's Database of Systematic Reviews were searched from database inception to April 22, 2020, supplemented by searches in health technology assessment websites. The International Society for Pharmacoeconomics and Outcomes Research (ISPOR) checklist covering six domains (relevance, credibility, analysis, reporting quality and transparency, interpretation and conflict of interest) was selected for appraisal of the included MA/NMA studies.
\end{abstract}

Results: Fifty-two full publications (36 MAs, 15 NMAs, and 1 MA/NMA) in PAH met the inclusion criteria. The majority of studies were of low quality, with none of the studies being scored as 'strong' across all checklist domains. Key limitations included the lack of a clearly defined, relevant decision problem, shortcomings in assessing and addressing between-study heterogeneity, and an incomplete or misleading interpretation of results.

Conclusions: This is the first critical appraisal of published MA/NMA studies in PAH, suggesting low quality and validity of published evidence synthesis studies in this therapeutic area. Besides the need for direct treatment comparisons assessed in long-term randomized controlled trials, future efforts in evidence synthesis in PAH should improve analysis quality and scrutiny in order to meaningfully address challenges arising from an evolving therapeutic landscape.

Keywords: Pulmonary hypertension, Evidence synthesis, Quality appraisal, Literature reviews, Meta-analysis, Network meta-analysis

\section{Background}

Pulmonary arterial hypertension (PAH) is a rare and debilitating chronic disease of the pulmonary vasculature [1]. Disease progression is characterized by increasing

* Correspondence: max.schlueter@iqvia.com

${ }^{1}$ IQVIA, 210 Pentonville Road, London N1 9JY, UK

Full list of author information is available at the end of the article pulmonary vascular resistance (PVR) and non-specific symptoms (e.g., dyspnoea during exercise, fatigue, chest pain, and light-headedness), that ultimately leads to right heart failure and premature death $[1,2]$. Prior to the availability of PAH-specific therapies, median survival time was documented as 2.8 years in the US patients

(c) The Author(s). 2020 Open Access This article is licensed under a Creative Commons Attribution 4.0 International License, which permits use, sharing, adaptation, distribution and reproduction in any medium or format, as long as you give appropriate credit to the original author(s) and the source, provide a link to the Creative Commons licence, and indicate if changes were made. The images or other third party material in this article are included in the article's Creative Commons licence, unless indicated otherwise in a credit line to the material. If material is not included in the article's Creative Commons licence and your intended use is not permitted by statutory regulation or exceeds the permitted use, you will need to obtain permission directly from the copyright holder. To view a copy of this licence, visit http://creativecommons.org/licenses/by/4.0/ The Creative Commons Public Domain Dedication waiver (http://creativecommons.org/publicdomain/zero/1.0/) applies to the data made available in this article, unless otherwise stated in a credit line to the data. 
with PAH [3]. Five-year survival rate in newly diagnosed patients is reported to be $61.2 \%$ [4].

Therapies in PAH have been approved with one or more routes of administration for three key pathogenesis pathways. Approved therapies targeting the nitric oxide pathway are the phosphodiesterase-5 inhibitors (PDE5I): sildenafil (oral or intravenous [IV]) and tadalafil (oral), and the soluble guanylate cyclase stimulator (sGCS) riociguat (oral). Therapies targeting the endothelin pathway currently approved are macitentan, bosentan and ambrisentan, all administered orally. One of the endothelin receptor antagonist (ERA) drugs, sitaxentan, was authorised in Europe in 2006, but subsequently withdrawn due to liver toxicity [5]. Approved drugs targeting the prostacyclin [PGI2] pathway include epoprostenol (IV), iloprost (inhaled), treprostinil (IV, inhaled, oral, subcutaneous $[\mathrm{SC}]$ ), beraprost (oral), and selexipag (oral), a selective non-prostanoid PGI2 receptor (IP receptor) agonist.

The treatment of PAH is guided by an evidence-based treatment algorithm published by the European Society of Cardiology and European Respiratory Society (ESC/ ERS) [2]. The overall treatment goal is to achieve a lowrisk status, associated with World Health Organization (WHO) Functional Class II, and good exercise capacity ( $>440 \mathrm{~m}$ in the 6 -min walking distance test), and rightventricular function assessed using echocardiography. The latest guidance and proceedings (see Figure S1 in the electronic supplementary material) recommend either monotherapy or initial oral combination therapy for treatment-naïve patients at a low or intermediate risk of clinical worsening or death $[2,6]$. For these patients, oral therapies are recommended, therefore ERA and PDE-5I are generally used as first-line treatment. For patients who fail to achieve an adequate clinical response (i.e. a low-risk status after 3 to 6 months) with initial therapy, treatment with sequential double or triple combination therapy is recommended. For high-risk treatment-naïve patients, an initial combination therapy regimen including a drug targeting the PGI2 pathway requiring continuous IV administration is indicated.

A lack of head-to-head treatment comparisons in randomized controlled trials (RCTs) has compounded clinical decision-making in PAH. As a result, a multitude of meta-analyses (MA; the synthesis of evidence from the same treatment comparisons assessed in clinical trials [7]) and network meta-analyses (NMA; the synthesis of evidence from both direct and indirect evidence to allow treatment comparisons that have not been directly assessed in clinical trials [7]) in PAH have been conducted.

Given the absence of direct RCT comparisons and the evolution of disease definition, classification, trials designs, available therapies and treatment guidelines, it is important to better understand the quality of published MA and NMA in PAH and their alignment with clinical decision-making today. The objective of the study was to critically appraise the quality and validity of published MA and NMA studies in PAH and explore the impact of the findings from these studies on current decisionmaking.

\section{Methods}

\section{Search strategy and data collection}

A systematic literature review was conducted according to the recommendations of the Cochrane Collaboration [8] and the Preferred Reporting Items for Systematic Reviews and Meta-Analyses (PRISMA) guidelines [9], to identify published evidence synthesis (i.e. MA and NMA) studies in PAH.

Searches were conducted from the database inception to September 12, 2018 and updated on April 22, 2020 in Embase, Medline (including Medline-In-Process) and the Cochrane's Database of Systematic Reviews via OVID in line with The National Institute for Health and Care Excellence (NICE) technology appraisal guidelines and recommendation from Centre for Review and Dissemination and the Cochrane Collaboration [10-12]. Supplementary searches included websites of selected health technology assessment agencies.

Retrieved records were assessed by one reviewer against the pre-specified PICOS criteria (Table S1 in the electronic supplementary material) and unblinded assessments were double checked by the second reviewer. Any discrepancies were resolved through discussion with a third reviewer. Studies were included if they met the following criteria: 1) adult patients with any etiology of $\mathrm{PAH}$ (pulmonary hypertension (PH) Group 1) [2], 2) at least two approved and available therapies or drug classes for treatment of PAH (to allow assessment of relative efficacy and safety of compared treatments), 3) full-text MA/NMA report. Details of the search methodology are provided in Tables S2a-h in the electronic supplementary material.

Key baseline characteristics of patients with PAH from the included RCTs were extracted to explore the extent of heterogeneity across the trials.

\section{Study appraisal}

A targeted review of published checklists for evidence synthesis studies was conducted. Checklists published by NICE [13], ISPOR [14], PRISMA [15] and GRADE [16] were identified. Criteria for checklist selection included:

- Domains covered, such as relevance of research question, methods for establishing the evidence base, assessment for internal validity, statistical methods, and reporting of results 
- Suitability to present context, including applicability to different forms of evidence synthesis

- Generalizability

- Acceptability and recognition of the checklist

The ISPOR checklist was deemed the most appropriate as it covers all domains listed in the checklist selection criteria, is suited to the study objective and is applicable to different types of evidence synthesis.

Complementary questions were added to the 26-item ISPOR checklist with questions specific to the disease area and/or study objective. These additional questions are marked as such in the study assessment provided in Table S3 in the electronic supplementary material.

The ISPOR checklist provides for a quality grading whereby an overall assessment of 'strong', 'neutral' or 'weak' is given for each of the six domains (i.e. relevance, credibility, analysis, reporting quality \& transparency, interpretation, conflict of interest). However, no explicit criteria are provided for scoring each domain. A set of criteria specific to each domain for quality grading was therefore adopted which is described in Table 1. Study appraisals by one reviewer were double checked by a second reviewer.

\section{Results}

\section{Study characteristics}

A total of $52 \mathrm{MA}$ and NMA studies met the inclusion criteria and were retained for data extraction and quality appraisal. From electronic database searches, 51 full-text publications were included. From the hand-search of publicly available websites of health technology assessment bodies, one report of the Canadian Agency for Drugs and Technologies in Health was included. The PRISMA diagram in Figure S2a-b (see electronic supplementary material) presents the search results.

The study characteristics of 52 publications included for appraisal are presented in Table 2. The publication year ranged between 2007 [44] and 2020 [39, 41, 49, 50] with most studies published in recent years. MAs were conducted in 35 studies $[17,19,20,22,23,26-29,31$, 35-41, 44-48, 51-53, 55, 58, 60-62, 65-69], NMAs in 15 studies $[18,21,24,25,30,32,33,42,49,50,54,56$, $59,63,64]$, both NMA and MA in one study [57], and MA and disproportionality analysis in one study [34]. Of 52 studies, 47 evaluated the impact of PAH interventions in patients with $\mathrm{PAH}$ and $\mathrm{PAH}$ subgroups (based on aetiology, e.g. idiopathic $\mathrm{PAH}$, familial PAH, connective tissue disease-associated PAH). Patients with $\mathrm{PH}$ including $\mathrm{PAH}$ and non-PAH patients (e.g. $\mathrm{PH}$ due to left sided heart disease) were investigated in four studies [20, 34, 44, 45] while patients with PAH were examined alongside other diseases (e.g. heart failure, prostate cancer) in two studies $[46,60]$.
Baseline characteristics of patient populations in the included studies are presented in Fig. 1a-c. The average WHO Functional Class distribution, a measure of disease severity, was $0.6,30.3,63.7$ and $5.4 \%$ for FC I, FC II, FC III and FC IV, respectively.

With a number of exceptions [17, 20, 24, 25, 34, 35, $39-41,46,48,56,60,61,63,64,66]$, most studies investigated treatments targeting all three pathways. All the approved treatments (ERA, PDE-5Is, PRAs, prostacyclin and sGCS) were investigated in nine recent studies [27, 30, 38, 42, 47, 49, 50, 59, 65]. Some studies included treatments approved in limited markets such as beraprost $[38,40,49,50,52,53,59,63,67]$. In nine studies, drugs targeting one pathway only were investigated: prostacyclins in five studies $[40,48,61,63,66]$ and ERAs in four studies $[25,46,60,64]$. Fifteen studies [17, 20, $21,24,25,32,34,35,39,46,60,62,64,65,67]$ focused on oral treatments only. Besides the approved treatments, non-approved PAH treatments were included in seven studies: imatinib [52, 53, 56, 62], terbogrel [29, 62] and aspirin [52]. Despite being withdrawn in 2010, sitaxentan was assessed in four recent studies [25, 35, 59, 62]. Two studies omitted selexipag despite being approved at the time of study [30,54].

The outcomes evaluated included clinical, hemodynamics, health-related-quality-of -life (HRQoL) and safety. Frequently investigated clinical endpoints were 6MWD (as a standalone or within combined events) in 43 studies $[17,19,20,22-33,35,36,39-42$, $44,45,47-52,54-63,65-68]$ followed by mortality (allcause or disease-specific) in 37 studies [18-21, 23, 25$30,33,37,40-42,44-55,57,60-63,65-68]$, clinical worsening (standalone or in combined events) in 25 studies [18-21, 24-27, 30, 31, 33, 35, 37, 38, 42, 47, 54, $57,59,61,62,65-68]$ and WHO functional class improvement or deterioration in 24 studies [18-20, 24, 27, $29,31-33,35,37,40,42,44,45,47,48,55,57,59,60$, $63,65,67]$.

The most commonly employed tool for quality assessment was Cochrane's risk of bias tool, employed in 21 studies $[20,21,27,32-39,41,47-51,60,62,64]$ followed by Jadad scores used in 12 studies [17, 25, 26, $30,40,42,48,51,61,65-67]$. There was no mention of quality appraisal being conducted in 10 studies $[18,24$, $28,29,44,45,55,58,63,64]$.

\section{Quality appraisal}

The quality assessment of the included studies is summarized in Fig. 2 by overall judgement (strength, neutral, weakness) against each domain of the checklist and the number of studies scoring each judgement in each domain in Table 3. The detailed quality assessments are presented in Table S3 in the electronic supplementary material. 
Table 1 Criteria for scoring each domain in the checklist

\begin{tabular}{|c|c|c|c|}
\hline Domains & Weak & Neutral & Strong \\
\hline Relevance & $\begin{array}{l}\text { At least three of the six checklist items } \\
\text { suggested study shortcomings, for } \\
\text { example omission of relevant therapies in } \\
\text { the analysis, omission of relevant } \\
\text { outcomes for evidence synthesis, or } \\
\text { inclusion of patients outside the target } \\
\text { population. }\end{array}$ & $\begin{array}{l}\text { 1-2 checklist items were not addressed } \\
\text { satisfactorily; no or insufficient justification } \\
\text { for a particular analysis approach was } \\
\text { provided (e.g. inclusion of oral therapies } \\
\text { only without justification). }\end{array}$ & $\begin{array}{l}\text { All checklist items were appropriately } \\
\text { addressed. }\end{array}$ \\
\hline Credibility & $\begin{array}{l}\text { Information omitted or insufficient } \\
\text { information provided for at least three of } \\
\text { the nine checklist items, for examples, } \\
\text { omission of key databases in the SLR, } \\
\text { omission of a quality assessment of } \\
\text { included studies, or lack of identification of } \\
\text { imbalances in the distribution of key effect } \\
\text { modifiers prior to the analysis. }\end{array}$ & $\begin{array}{l}\text { 1-2 checklist items were not addressed } \\
\text { satisfactorily, for example, an adequate } \\
\text { search strategy but no transparent } \\
\text { reporting of the full search strings, or lack } \\
\text { of reporting of the results of the quality } \\
\text { assessment. }\end{array}$ & $\begin{array}{l}\text { All checklist item were addressed } \\
\text { appropriately. The checklist domain } \\
\text { 'credibility' includes one question only } \\
\text { applicable to NMA studies; this question } \\
\text { was not considered for the domain } \\
\text { grading of MA studies. }\end{array}$ \\
\hline Analysis & $\begin{array}{l}\text { At least three of the } 10 \text { checklist items } \\
\text { suggested study shortcomings, such as } \\
\text { lack of subgroup analyses or meta- } \\
\text { regression in cases of between-study het- } \\
\text { erogeneity, pooling of drug classes, treat- } \\
\text { ments or doses without proper } \\
\text { justification, or lack of a valid rationale for } \\
\text { the use of random effects or fixed effect } \\
\text { models. }\end{array}$ & $\begin{array}{l}1-2 \text { checklist items were not addressed } \\
\text { satisfactorily, such as insufficient detail on } \\
\text { the statistical model. }\end{array}$ & $\begin{array}{l}\text { All checklist items were addressed } \\
\text { appropriately. The checklist domain } \\
\text { 'analysis' includes four questions only } \\
\text { applicable to NMA studies; these questions } \\
\text { were not considered for the domain } \\
\text { grading of MA studies. }\end{array}$ \\
\hline $\begin{array}{l}\text { Reporting } \\
\text { quality \& } \\
\text { transparency }\end{array}$ & $\begin{array}{l}\text { At least two of the six checklist items were } \\
\text { not addressed satisfactorily, or discussion } \\
\text { of the impact of important patient } \\
\text { characteristics on treatment effects was } \\
\text { not included. }\end{array}$ & $\begin{array}{l}\text { Insufficient information for one checklist } \\
\text { item or a brief discussion of the impact of } \\
\text { the impact of patient characteristics on } \\
\text { analysis results was provided. }\end{array}$ & $\begin{array}{l}\text { All checklist items were addressed } \\
\text { appropriately. The checklist domain } \\
\text { 'reporting quality \& transparency' includes } \\
\text { four questions only applicable to NMA } \\
\text { studies; these questions were not } \\
\text { considered for the domain grading of MA } \\
\text { studies. }\end{array}$ \\
\hline Interpretation & $\begin{array}{l}\text { Results were not contextualized with } \\
\text { consideration of limitations or specific } \\
\text { treatments were endorsed over others } \\
\text { despite a lack of discussion of between- } \\
\text { study heterogeneity and/or despite pool- } \\
\text { ing of active therapies. }\end{array}$ & $\begin{array}{l}\text { Study limitations (e.g. between-study het- } \\
\text { erogeneity) were provided however with- } \\
\text { out a detailed discussion of the impact } \\
\text { these may have had on observed study } \\
\text { results. }\end{array}$ & $\begin{array}{l}\text { All these aspects were addressed } \\
\text { appropriately. }\end{array}$ \\
\hline $\begin{array}{l}\text { Conflict of } \\
\text { interest }\end{array}$ & $\begin{array}{l}\text { No information on conflicts of interest was } \\
\text { provided, or details of author disclosures } \\
\text { and contributions were insufficient. }\end{array}$ & $\begin{array}{l}\text { Disclosures as well as author contributions } \\
\text { were clearly stated in cases of personal or } \\
\text { financial relationships of affiliations that } \\
\text { could have biased the work in question. }\end{array}$ & $\begin{array}{l}\text { No personal or financial relationships or } \\
\text { affiliations (that could have biased the } \\
\text { study) were declared. }\end{array}$ \\
\hline
\end{tabular}

MA Meta-analysis, NMA Network meta-analysis, SLR Systematic literature review

\section{Relevance}

Of the 52 studies reviewed, eight were scored as strong in terms of relevance, 26 as neutral, and the remaining 18 as weak.

Most included studies included relevant populations. In some cases, the population was narrowly defined and thus not generalizable to an overall $\mathrm{PAH}$ population (e.g. focused on connective tissue disease-associated-PAH [36, 39]) while in others, it went beyond adult PAH populations (i.e. $\mathrm{PH}$ patients [group 2-5] or pediatric $\mathrm{PAH}$ were included). Some studies adopted a narrow research focus on 1-2 drug classes $[17,20,25,32,35,39,40,60,61,63$, $64,66]$ or oral therapies only $[17,20,21,32,35,39,62,64$, $65,67]$, often without explicit and/or adequate justification for such restrictions. Many included studies were highly selective in their choice of outcomes analyzed, 6MWD being the most frequently analyzed outcome.
Very few studies fulfilled the checklist item about the extent to which an evidence synthesis study is informative to decision makers today and aligned with the current clinical practice and guidelines. Several papers did not explicitly state the research question or decision problem guiding the analysis [18, 21, 29, 33, 42, 55, 61]. Several other studies failed to justify the focus or their research question $[17,18,20,21,25,31,32,40,45,60$, 62-66]. For example, some studies formulated research questions with a very narrow scope (e.g. oral treatments $[17,20,21,32,62,64,65])$ or included trials with nonPAH populations $[34,44,45]$, therefore precluding determination of the optimal choice of therapy based on a comparison of all available treatment options. Some studies included unapproved or withdrawn treatments, while several studies made conclusions at odds with current knowledge, guidelines and clinical practice. For 
Table 2 Characteristics of evidence synthesis studies

\begin{tabular}{|c|c|c|c|c|c|c|}
\hline $\begin{array}{l}\text { Study ID } \\
\text { (author year) }\end{array}$ & Patient population & $\begin{array}{l}\text { Type of evidence } \\
\text { synthesis }\end{array}$ & $\begin{array}{l}\text { Number of } \\
\text { studies } \\
\text { included }\end{array}$ & Treatments included & Outcomes included & $\begin{array}{l}\text { Quality assessment tool } \\
\text { (used for included trials) }\end{array}$ \\
\hline $\begin{array}{l}\text { Avouac } 2008 \\
{[17]}\end{array}$ & $\begin{array}{l}\text { Patients with PAH } \\
\text { (including idiopathic, } \\
\text { secondary to CTD or CHD) }\end{array}$ & MA & 10 & $\begin{array}{l}\text { Oral ERAs (bosentan, } \\
\text { sitaxentan) and PDE-5I (oral } \\
\text { sildenafil) }\end{array}$ & $6 \mathrm{MWD}$ & Jadad scores \\
\hline $\begin{array}{l}\text { Badiani } 2016^{\mathrm{a}} \\
{[18]}\end{array}$ & $\begin{array}{l}\text { Patients with PAH } \\
\text { (including associated PAH } \\
\text { and IPAH) }\end{array}$ & NMA & 17 & $\begin{array}{l}\text { Oral ERAs (bosentan, } \\
\text { ambrisentan and } \\
\text { macitentan), oral PDE-5Is } \\
\text { (sildenafil, tadalafil and var- } \text { denafil }^{\text {), }} \text {, prostanoids (oral } \\
\text { beraprost and oral treprosti- } \\
\text { nil) sPRA (oral selexipag), } \\
\text { sGCS (oral riociguat) }\end{array}$ & $\begin{array}{l}\text { Composite clinical } \\
\text { worsening }\end{array}$ & Not reported \\
\hline Bai 2011 [19] & Patients with PAH & MA & 6 & $\begin{array}{l}\text { Oral PDE-5Is (tadalafil and } \\
\text { sildenafil), ERA (bosentan), } \\
\text { prostanoids (inhaled ilo- } \\
\text { prost and IV epoprostenol) } \\
\text { developed and approved } \\
\text { for PAH; combination ther- } \\
\text { apies only included with } 2 \\
\text { or } 3 \text { drugs. }\end{array}$ & $\begin{array}{l}\text { 6MWD, clinical worsening, } \\
\text { NYHA FC, mPAP, RAP, PVR } \\
\text { and cardiac output, SAEs, } \\
\text { all-cause mortality }\end{array}$ & $\begin{array}{l}\text { Quality assessment } \\
\text { completed, tool not stated. }\end{array}$ \\
\hline $\begin{array}{l}\text { Barnes } 2019 \\
{[20]}\end{array}$ & $\begin{array}{l}\text { Patients with PH (all } \\
\text { groups 1-5) }\end{array}$ & MA & 3 (for $\mathrm{PAH}$ ) & $\begin{array}{l}\text { Oral PDE5Is (sildenafil, } \\
\text { tadalafil), oral ERAs } \\
\text { (ambrisentan, bosentan) }\end{array}$ & $\begin{array}{l}\text { Primary outcomes: WHO FC, } \\
6 \mathrm{MWD} \text { and mortality. } \\
\text { Secondary outcomes: } \\
\text { Haemodynamic parameters, } \\
\text { quality of life/health status, } \\
\text { dyspnoea, clinical } \\
\text { worsening (hospitalisation/ } \\
\text { intervention), and AEs }\end{array}$ & Cochrane's risk of bias \\
\hline $\begin{array}{l}\text { Biondi-Zoccai } \\
2013[21]\end{array}$ & Patients with PAH & NMA & 6 & $\begin{array}{l}\text { First line oral drugs: oral } \\
\text { ERAs (bosentan, sitaxentan, } \\
\text { ambrisentan, prostacyclin } \\
\text { analogues (oral beraprost), } \\
\text { oral PDE-5Is (tadalafil, } \\
\text { sildenafil) }\end{array}$ & $\begin{array}{l}\text { All-cause mortality, clinical } \\
\text { improvement and clinical } \\
\text { worsening }\end{array}$ & Cochrane's risk of bias \\
\hline $\begin{array}{l}\text { Chen } 2009 \\
{[22]}\end{array}$ & $\begin{array}{l}\text { Patients with PAH and } \\
\text { subgroups (i.e. idiopathic } \\
\text { PAH, CTD-associated PAH) }\end{array}$ & MA & 20 & $\begin{array}{l}\text { Any of epoprostenol (IV), } \\
\text { iloprost (inhaled), bosentan } \\
\text { (oral), sitaxentan (oral) and } \\
\text { sildenafil (oral) }\end{array}$ & $\begin{array}{l}\text { Survival, time to clinical } \\
\text { deterioration, HRQoL, } \\
6 \mathrm{MWD} \text {, symptomatic } \\
\text { improvement, frequency } \\
\text { and duration of } \\
\text { hospitalization and } \\
\text { outpatient/GP visits, SAEs, } \\
\text { AEs, withdrawal, } \\
\text { haemodynamic assessment }\end{array}$ & $\begin{array}{l}\text { Quality assessment } \\
\text { completed, tool not stated }\end{array}$ \\
\hline $\begin{array}{l}\text { Coeytaux } 2014 \\
\text { [23] [McCrory } \\
2013 \text { full } \\
\text { report] }\end{array}$ & Patients with $\mathrm{PAH}$ & NMA & 28 & $\begin{array}{l}\text { Oral PDE-5I (sildenafil and } \\
\text { tadalafil), oral ERAs (bosen- } \\
\text { tan and ambrisentan), pros- } \\
\text { tanoids (IV epoprostenol, } \\
\text { inhaled iloprost and IV or } \\
\text { SC treprostinil) and calcium } \\
\text { channel blockers }\end{array}$ & $\begin{array}{l}\text { Mortality, } 6 \mathrm{MWD} \text {, } \\
\text { hospitalization, } \\
\text { hemodynamic measures } \\
\text { (i.e. PVR, PAP, cardiac index), } \\
\text { and commonly reported } \\
\text { AEs. }\end{array}$ & $\begin{array}{l}\text { Quality appraisal approach } \\
\text { as described in the US } \\
\text { Agency for Healthcare } \\
\text { Research and Quality's } \\
\text { "Methods Guide for } \\
\text { Effectiveness and } \\
\text { Comparative Effectiveness } \\
\text { Reviews. }\end{array}$ \\
\hline $\begin{array}{l}\text { Dranitsaris } \\
2009[24]\end{array}$ & Patients with PAH & MA & 9 & $\begin{array}{l}\text { Oral treatments: } \\
\text { ambrisentan, bosentan, } \\
\text { sitaxentan and sildenafil }\end{array}$ & $\begin{array}{l}6 \mathrm{MWD}, \mathrm{BDI}, \mathrm{NYHA} \\
\text { Functional Class and clinical } \\
\text { worsening }\end{array}$ & Not reported \\
\hline $\begin{array}{l}\text { Duo-Ji } 2017 \\
{[25]}\end{array}$ & $\begin{array}{l}\text { Patients with symptomatic } \\
\text { PAH, idiopathic PAH or } \\
\text { PAH associated with other } \\
\text { diseases }\end{array}$ & NMA & 10 & $\begin{array}{l}\text { Oral ERAs only } \\
\text { (ambrisentan, bosentan, } \\
\text { sitaxentan and macitentan) }\end{array}$ & $\begin{array}{l}6 \mathrm{MWD} \text {, clinical worsening, } \\
\mathrm{SAE}, \text { mortality and all-cause } \\
\text { discontinuation }\end{array}$ & Jadad scores \\
\hline Fox $2011^{b}[26]$ & $\begin{array}{l}\text { Patients with PAH } \\
\text { (including idiopathic PAH, } \\
\text { familial PAH, CTD } \\
\text { associated PAH, } \\
\text { pulmonary shut, portal } \\
\text { hypertension, HIV } \\
\text { infection and thyroid } \\
\text { disease) }\end{array}$ & MA & 6 & $\begin{array}{l}\text { Oral PDE-5I (sildenafil and } \\
\text { tadalafil, ERA (oral bosen- } \\
\text { tan), prostanoids (IV epo- } \\
\text { prostenol, inhaled iloprost } \\
\text { and inhaled treprostinil de- } \\
\text { veloped and approved for } \\
\text { PAH }\end{array}$ & $\begin{array}{l}6 \mathrm{MWD} \text {, clinical worsening, } \\
\text { mortality, hospitalization for } \\
\text { PAH deterioration, lung } \\
\text { transplantation, escalation } \\
\text { of treatment and safety } \\
\text { outcomes }\end{array}$ & Jadad scores \\
\hline Fox 2016 [27] & Patients with PAH & MA & 18 & $\begin{array}{l}\text { Prostanoids (IV } \\
\text { epoprostenol, inhaled } \\
\text { iloprost and inhaled/oral } \\
\text { treprostinil) oral ERAs }\end{array}$ & $\begin{array}{l}\text { Primary outcomes: all-cause } \\
\text { mortality (analysed separ- } \\
\text { ately) and composite clin- } \\
\text { ical worsening. Secondary }\end{array}$ & Cochrane's risk of bias \\
\hline
\end{tabular}


Table 2 Characteristics of evidence synthesis studies (Continued)

\begin{tabular}{|c|c|c|c|c|c|c|}
\hline $\begin{array}{l}\text { Study ID } \\
\text { (author year) }\end{array}$ & Patient population & $\begin{array}{l}\text { Type of evidence } \\
\text { synthesis }\end{array}$ & $\begin{array}{l}\text { Number of } \\
\text { studies } \\
\text { included }\end{array}$ & Treatments included & Outcomes included & $\begin{array}{l}\text { Quality assessment tool } \\
\text { (used for included trials) }\end{array}$ \\
\hline & & & & $\begin{array}{l}\text { (bosentan, ambrisentan, } \\
\text { sitaxsentan and } \\
\text { macitentan), oral PDE-5I (sil- } \\
\text { denafil and tadalafil), sGCS } \\
\text { (oral riociguat), sPRA (oral } \\
\text { selexipag) with their ap- } \\
\text { proved dose }\end{array}$ & $\begin{array}{l}\text { outcomes: 6MWD, PAP, car- } \\
\text { diac index, WHO Functional } \\
\text { Class. }\end{array}$ & \\
\hline $\begin{array}{l}\text { Gabler } 2012 \\
{[28]}\end{array}$ & $\begin{array}{l}\text { Patients with PAH } \\
\text { (including idiopathic PAH, } \\
\text { CTD-associated PAH, CHD- } \\
\text { associated PAH, HIV } \\
\text { infection) }\end{array}$ & MA & 10 & $\begin{array}{l}\text { Oral PDE-5I (sildenafil and } \\
\text { tadalafil), oral ERAs (ambri- } \\
\text { sentan, bosentan and sitax- } \\
\text { entan) and prostanoids } \\
\text { (inhaled iloprost and SC } \\
\text { treprostinil) }\end{array}$ & $\begin{array}{l}6 \mathrm{MWD} \text {, mortality, lung } \\
\text { transplantation, atrial } \\
\text { septostomy, hospitalization } \\
\text { due to PAH worsening, } \\
\text { withdrawal for worsening } \\
\text { right-sided heart failure, or } \\
\text { addition of other PAH } \\
\text { medications }\end{array}$ & Not reported \\
\hline $\begin{array}{l}\text { Galie 2009b } \\
\text { [29] }\end{array}$ & Patients with PAH & MA & 21 & $\begin{array}{l}\text { Both approved and not } \\
\text { approved treatments for } \\
\text { PAH (oral ambrisentan, oral } \\
\text { bosentan, oral sitaxentan, } \\
\text { oral sildenafil, inhaled } \\
\text { iloprost, oral beraprost, IV } \\
\text { epoprostenol, SC } \\
\text { treprostinil, oral terbogrel }^{d} \text { ) }\end{array}$ & $\begin{array}{l}\text { Primary outcome: all-cause } \\
\text { mortality Secondary out- } \\
\text { comes: PAH-related hospi- } \\
\text { talizations to PAH, 6MWD, } \\
\text { NYHA WHO Functional } \\
\text { Class, RAP, PAP, cardiac } \\
\text { index, and PVR }\end{array}$ & Not reported \\
\hline Gao 2017 [30] & Patients with PAH & NMA & 32 & $\begin{array}{l}\text { Prostanoids (IV } \\
\text { epoprostenol, inhaled } \\
\text { iloprost, oral beraprost and } \\
\text { oral/inhaled/SC treprostinil), } \\
\text { oral ERAs (bosentan, } \\
\text { ambrisentan and } \\
\text { macitentan), oral PDE-5Is } \\
\text { (sildenafil, tadalafil and var- }^{\text {denafil }} \text { ), sGCS (oral rioci- } \\
\text { guat), and combination } \\
\text { therapy regardless of drug } \\
\text { dosage forms }\end{array}$ & $\begin{array}{l}\text { Primary endpoint: } 6 \mathrm{MWD} \\
\text { Secondary endpoints: PAP, } \\
\text { PVR, all-cause mortality, and } \\
\text { composite clinical worsen- } \\
\text { ing. Safety endpoint: SAEs }\end{array}$ & Jadad scores \\
\hline He 2010 [31] & Patients with PAH & MA & 11 & $\begin{array}{l}\text { Oral bosentan, oral } \\
\text { sildenafil and inhaled } \\
\text { iloprost }\end{array}$ & $\begin{array}{l}\text { Clinical worsening, NYHA/ } \\
\text { WHO Functional Class, } \\
6 \mathrm{MWD} \text {, and hemodynamic } \\
\text { parameters including } \\
\text { systolic PAP, PAP, PVR, } \\
\text { cardiac output and cardiac } \\
\text { index, treatment-related } \\
\text { SAEs. }\end{array}$ & Juni scale \\
\hline $\begin{array}{l}\text { lgarashi } 2016 \\
{[32]}\end{array}$ & Patients with PAH & NMA & 7 & $\begin{array}{l}5 \text { oral PAH treatments: } \\
\text { ambrisentan, bosentan, } \\
\text { sildenafil, tadalafil, and } \\
\text { beraprost }\end{array}$ & $\begin{array}{l}6 \mathrm{MWD}, \text { WHO Functional } \\
\text { Class and PAP }\end{array}$ & Cochrane's risk of bias \\
\hline Jain 2017 [33] & $\begin{array}{l}\text { Patients with symptomatic } \\
\text { PAH }\end{array}$ & NMA & 31 & $\begin{array}{l}\text { All US-FDA approved PAH- } \\
\text { specific drugs: oral ERAs } \\
\text { (bosentan, ambrisentan and } \\
\text { macitentan), oral PDE-5Is } \\
\text { (sildenafil and tadalafil), } \\
\text { prostanoids (oral/inhaled/ } \\
\text { SC/IV treprostinil, inhaled } \\
\text { iloprost and IV epoproste- } \\
\text { nol), sGCS (oral riociguat) } \\
\text { and sPRA (oral selexipag) }\end{array}$ & $\begin{array}{l}\text { Primary efficacy outcome: } \\
\text { composite clinical } \\
\text { worsening } \\
\text { Secondary efficacy } \\
\text { outcomes: PAH-related } \\
\text { hospitalization and all-cause } \\
\text { mortality Safety outcome: } \\
\text { treatment-related AEs lead- } \\
\text { ing to drug discontinuation }\end{array}$ & Cochrane's risk of bias \\
\hline $\begin{array}{l}\text { Khouri } 2018 \\
{[34]}\end{array}$ & $\begin{array}{l}\text { Patients with } \mathrm{PH} \text { in the } \\
\text { main analysis; patients } \\
\text { with PAH in the sensitivity } \\
\text { analysis }\end{array}$ & $\begin{array}{l}\text { MA and a } \\
\text { disproportionality } \\
\text { analysis }\end{array}$ & $\begin{array}{l}13 \text { (7 in } \mathrm{PAH} \\
\text { patients) }\end{array}$ & $\begin{array}{l}\text { Oral PDE-5Is (sildenafil and } \\
\text { tadalafil) and sGCS (oral } \\
\text { riociguat) }\end{array}$ & AEs & $\begin{array}{l}\text { Cochrane's risk of bias and } \\
\text { GRADE for evidence }\end{array}$ \\
\hline $\begin{array}{l}\text { Kirtania } 2019 \\
{[35]}\end{array}$ & $\begin{array}{l}\text { Patients with PAH of any } \\
\text { aetiology }\end{array}$ & MA & 7 & $\begin{array}{l}\text { Combination of oral ERAs } \\
\text { (ambrisentan, bosentan, } \\
\text { macitentan, sitaxentan) } \\
\text { with oral PDE5Is (sildenafil } \\
\text { or tadalafil), ERA or PDEI } \\
\text { monotherapies }\end{array}$ & $\begin{array}{l}\text { Primary outcome: } 6 \mathrm{MWD} \\
\text { Secondary outcomes: } \\
\text { Clinical worsening (death, } \\
\text { hospitalisation, WHO FC, } \\
\text { lung transplantation, clinical } \\
\text { deterioration of PAH } \\
\text { requiring additional } \\
\text { therapy, PVR and NT- } \\
\text { proBNP }\end{array}$ & Cochrane's risk of bias \\
\hline
\end{tabular}


Table 2 Characteristics of evidence synthesis studies (Continued)

\begin{tabular}{|c|c|c|c|c|c|c|}
\hline $\begin{array}{l}\text { Study ID } \\
\text { (author year) }\end{array}$ & Patient population & $\begin{array}{l}\text { Type of evidence } \\
\text { synthesis }\end{array}$ & $\begin{array}{l}\text { Number of } \\
\text { studies } \\
\text { included }\end{array}$ & Treatments included & Outcomes included & $\begin{array}{l}\text { Quality assessment tool } \\
\text { (used for included trials) }\end{array}$ \\
\hline $\begin{array}{l}\text { Kuwana } 2013 \\
{[36]}\end{array}$ & $\begin{array}{l}\text { Patients with PAH and } \\
\text { CTD-associated PAH }\end{array}$ & MA & 19 & $\begin{array}{l}\text { Oral PDE-5I (sildenafil and } \\
\text { tadalafil), oral ERAs (bosen- } \\
\text { tan and ambrisentan), pros- } \\
\text { tacyclin analogues (IV } \\
\text { epoprostenol, oral bera- } \\
\text { prost, inhaled iloprost and } \\
\text { IV/SC/inhaled treprostinil) }\end{array}$ & $6 \mathrm{MWD}$ & Cochrane's risk of bias \\
\hline $\begin{array}{l}\text { Lajoie } 2016 \\
\text { [37] }\end{array}$ & $\begin{array}{l}\text { Patients with PAH } \\
\text { (including idiopathic PAH, } \\
\text { associated PAH, or } \\
\text { hereditary PAH) }\end{array}$ & MA & 17 & $\begin{array}{l}\text { Prostanoids (IV } \\
\text { epoprostenol, inhaled/oral } \\
\text { treprostinil, inhaled } \\
\text { iloprost), oral ERAs } \\
\text { (bosentan, ambrisentan and } \\
\text { macitentan), oral PDE-5I (sil- } \\
\text { denafil, tadalafil and varde- } \\
\text { nafil }^{\text {d) }} \text { or sGCS (oral } \\
\text { riociguat) }^{\text {ingal }}\end{array}$ & $\begin{array}{l}\text { Primary outcome: clinical } \\
\text { worsening Secondary } \\
\text { outcomes: all-cause mortal- } \\
\text { ity, PAH-related mortality, } \\
\text { PAH-related hospitalizations, } \\
\text { lung transplantation, treat- } \\
\text { ment escalation, symptom- } \\
\text { atic progression, WHO } \\
\text { Functional Class, exercise } \\
\text { capacity, treatment discon- } \\
\text { tinuation, and treatment } \\
\text { duration }\end{array}$ & Cochrane's risk of bias \\
\hline $\begin{array}{l}\text { Lajoie } 2018 \\
{[38]}\end{array}$ & $\begin{array}{l}\text { Patients with PAH } \\
\text { (including idiopathic PAH } \\
\text { and associated PAH) }\end{array}$ & MA & 15 & $\begin{array}{l}\text { Currently licensed PAH- } \\
\text { specific therapies: prosta- } \\
\text { noids (IV epoprostenol, in- } \\
\text { haled iloprost, inhaled/oral } \\
\text { treprostinil), oral ERAs } \\
\text { (ambrisentan, bosentan, } \\
\text { and macitentan), oral PDE- } \\
\text { 5Is (sildenafil, tadalafil, and } \\
\text { vardenafil }^{d} \text { ), sGCS (oral rioci- } \\
\text { guat), and a sPRA (oral } \\
\text { selexipag) }\end{array}$ & Clinical worsening & Cochrane's risk of bias \\
\hline Lei 2020 [39] & $\begin{array}{l}\text { Patients with CTD- } \\
\text { associated PAH or SSC- } \\
\text { PAH }\end{array}$ & MA & 27 & $\begin{array}{l}\text { Combination of oral ERAs } \\
\text { (ambrisentan, bosentan) } \\
\text { with oral PDE5Is (sildenafil } \\
\text { or tadalafil), oral ERA or oral } \\
\text { PDEI monotherapies }\end{array}$ & $\begin{array}{l}\text { 6MWD, hemodynamics } \\
\text { parameters (PVR, PAP) not } \\
\text { analysed due to insufficient } \\
\text { data }\end{array}$ & Cochrane's risk of bias \\
\hline Li 2013 [40] & Patients with PAH & MA & 14 & $\begin{array}{l}\text { Prostanoids (IV } \\
\text { epoprostenol, inhaled } \\
\text { iloprost, SC/inhaled } \\
\text { treprostinil, oral beraprost) }\end{array}$ & $\begin{array}{l}\text { Efficacy or safety endpoints } \\
\text { (e.g. 6MWD, NYHA } \\
\text { Functional Class, PAP, PVR, } \\
\text { or all-cause mortality) }\end{array}$ & Jadad scores \\
\hline Li 2020 [41] & Patients with $\mathrm{PAH}$ & NMA & 9 & $\begin{array}{l}\text { Oral ambrisentan, oral } \\
\text { bosentan, oral sildenafil }\end{array}$ & $\begin{array}{l}\text { 6MWD, PAP, cardiac index, } \\
\text { PVR, RAP and mortality }\end{array}$ & $\begin{array}{l}\text { Cochrane's risk of bias and } \\
\text { Jadad score }\end{array}$ \\
\hline Lin 2018 [42] & Patients with PAH & NMA & 43 & $\begin{array}{l}\text { Oral ERAs (bosentan, } \\
\text { macitentan, sitaxentan and } \\
\text { ambrisentan) sGCS (oral } \\
\text { riociguat), oral PDE-5Is (sil- } \\
\text { denafil, tadalafil and varde- } \\
\text { nafil }^{d} \text { ), Prostanoids (IV } \\
\text { epoprostenol, IV/inhaled/ } \\
\text { oral/SC treprostinil, inhaled } \\
\text { iloprost and oral beraprost), } \\
\text { and sPRA (oral selexipag) } \\
\text { monotherapy or in } \\
\text { combination }\end{array}$ & $\begin{array}{l}\text { 6MWD, Functional Class } \\
\text { amelioration, mortality, } \\
\text { clinical worsening, SAEs, } \\
\text { withdrawal, PVR, PAP, } \\
\text { cardiac index, and RAP }\end{array}$ & Jadad scores \\
\hline Liu 2016 [43] & Patients with PAH & MA & 35 & $\begin{array}{l}\text { Prostanoids (IV } \\
\text { epoprostenol, inhaled/IV/ } \\
\text { SC/oral treprostinil, inhaled } \\
\text { iloprost, oral beraprost and } \\
\text { oral selexipag), oral ERAs } \\
\text { (bosentan, ambrisentan and } \\
\text { macitentan), oral PDE-5I (sil- } \\
\text { denafil, tadalafil and varde- } \\
\text { nafil }^{d} \text { ) sGCSs (oral riociguat) } \\
\text { and rho-kinase inhibitor }_{\text {(fasudil }} \text {; ROA unclear) }\end{array}$ & $\begin{array}{l}\text { Primary outcomes: } \\
\text { Mortality, 6MWD, WHO/ } \\
\text { NYHA Functional Class } \\
\text { Secondary outcomes: } \\
\text { Cardiopulmonary } \\
\text { hemodynamics including } \\
\text { PAP, PVR, cardiac index, } \\
\text { withdrawal due to AEs }\end{array}$ & Cochrane's risk of bias \\
\hline $\begin{array}{l}\text { Macchia } 2007 \\
{[44]}\end{array}$ & $\begin{array}{l}\text { Patient with } \mathrm{PH} \text { (including } \\
\text { primary } \mathrm{PH} \text { due to CTD } \\
\text { and } \mathrm{PH} \text { related to } \\
\text { thromboembolic disease) }\end{array}$ & MA & 16 & $\begin{array}{l}\text { Prostanoids (IV } \\
\text { epoprostenol, SC } \\
\text { treprostinil, inhaled iloprost } \\
\text { and oral beraprost), oral } \\
\text { ERAs (sitaxentan and } \\
\text { bosentan, and PDE-5I (oral }\end{array}$ & $\begin{array}{l}\text { Total mortality, NYHA } \\
\text { Functional Class and 6MWD }\end{array}$ & Not reported \\
\hline
\end{tabular}


Table 2 Characteristics of evidence synthesis studies (Continued)

\begin{tabular}{|c|c|c|c|c|c|c|}
\hline $\begin{array}{l}\text { Study ID } \\
\text { (author year) }\end{array}$ & Patient population & $\begin{array}{l}\text { Type of evidence } \\
\text { synthesis }\end{array}$ & $\begin{array}{l}\text { Number of } \\
\text { studies } \\
\text { included }\end{array}$ & Treatments included & Outcomes included & $\begin{array}{l}\text { Quality assessment tool } \\
\text { (used for included trials) }\end{array}$ \\
\hline & & & & \multicolumn{3}{|l|}{ sildenafil) } \\
\hline $\begin{array}{l}\text { Macchia } 2010 \\
{[45]}\end{array}$ & $\begin{array}{l}\text { Patients with } \mathrm{PH} \\
\text { (including idiopathic PAH } \\
\text { and PAH-related } \\
\text { conditions) }\end{array}$ & MA & 26 & $\begin{array}{l}\text { Prostanoids (inhaled } \\
\text { iloprost, SC treprostinil and } \\
\text { IV epoprostenol), oral ERAs } \\
\text { (bosentan, ambrisentan and } \\
\text { sitaxentan), and oral PDE-5I } \\
\text { (sildenafil and tadalafil) }\end{array}$ & $\begin{array}{l}\text { Total mortality, NYHA } \\
\text { Functional Class and 6MWD }\end{array}$ & Not reported \\
\hline Pan 2017 [46] & $\begin{array}{l}\text { Patients with different } \\
\text { diseases including PAH }\end{array}$ & MA & 33 & $\begin{array}{l}\text { All oral ERAs (atrasentan }{ }^{\mathrm{d}} \text {, } \\
\text { avosentan }^{\mathrm{d}} \text {, ambrisentan, } \\
\text { bosentan, darusentan }{ }^{\mathrm{d}} \text {, } \\
\text { macitentan, sitaxentan and } \\
\text { zibotentan }^{\text {d) }}\end{array}$ & $\begin{array}{l}\text { Mortality, CVD increased } \\
\text { risk, AEs }\end{array}$ & $\begin{array}{l}\text { The Newcastle-Ottawa } \\
\text { scale }\end{array}$ \\
\hline Pan 2018 [47] & $\begin{array}{l}\text { Patients with CTD- } \\
\text { associated PAH only }\end{array}$ & MA & 6 & 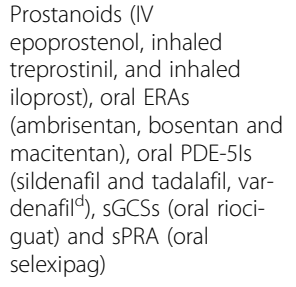 & $\begin{array}{l}\text { Primary outcome: } \\
\text { composite clinical } \\
\text { worsening } \\
\text { Secondary outcomes: } \\
6 \mathrm{MWD} \text {, N-terminal pro-B } \\
\text { type natriuretic peptide } \\
\text { (NT-proBNP), WHO/NYHA } \\
\text { Functional Class or cardio- } \\
\text { pulmonary hemodynamics }\end{array}$ & Cochrane's risk of bias \\
\hline $\begin{array}{l}\text { Paramothayan } \\
2009 \text { [48] }\end{array}$ & $\begin{array}{l}\text { Patients with primary } \mathrm{PH} \\
\text { and its variant }\end{array}$ & MA & 9 & $\begin{array}{l}\text { Prostanoids (IV/inhaled } \\
\text { lloprost, IV epoprostenol, IV/ } \\
\text { SC/oral treprostinil and oral } \\
\text { beraprost) }\end{array}$ & $\begin{array}{l}\text { Primary outcomes: 6MWD } \\
\text { NYHA Functional Class } \\
\text { Secondary outcomes: } \\
\text { Mortality and AEs }\end{array}$ & $\begin{array}{l}\text { The Cochrane approach } \\
\text { and the Jadad score }\end{array}$ \\
\hline $\begin{array}{l}\text { Petrovic 2020a } \\
\text { [49] }\end{array}$ & Patients with $\mathrm{PAH}$ & NMA & 16 & $\begin{array}{l}\text { Oral ERAs (ambrisentan, } \\
\text { bosentan, macitentan), oral } \\
\text { PDE5Is (sildenafil, tadalafil), } \\
\text { prostanoids (IV } \\
\text { epoprostenol, oral/inhaled } \\
\text { treprostinil, inhaled iloprost, } \\
\text { oral beraprost), sGCSs (oral } \\
\text { riociguat), sPRA (oral } \\
\text { selexipag) as add-on } \\
\text { therapies }\end{array}$ & $\begin{array}{l}6 \mathrm{MWD} \text {, all-cause mortality, } \\
\text { discontinuation due to AEs }\end{array}$ & Cochrane's risk of bias \\
\hline $\begin{array}{l}\text { Petrovic 2020b } \\
{[50]}\end{array}$ & Patients with PAH & NMA & 21 & $\begin{array}{l}\text { Oral ERAs (ambrisentan, } \\
\text { bosentan, macitentan), oral } \\
\text { PDE5Is (sildenafil, tadalafil), } \\
\text { prostanoids (IV } \\
\text { epoprostenol, SC } \\
\text { treprostinil, inhaled iloprost, } \\
\text { oral beraprost), sGCSs (oral } \\
\text { riociguat), sPRA (oral } \\
\text { selexipag) }\end{array}$ & $\begin{array}{l}\text { Efficacy outcomes: } 6 \mathrm{MWD} \text {, } \\
\text { all-cause mortality } \\
\text { Safety outcome: } \\
\text { discontinuation due to AEs }\end{array}$ & Cochrane's risk of bias \\
\hline $\begin{array}{l}\text { Ryerson } 2010 \\
{[51]}\end{array}$ & Patients with PAH & MA & 24 & $\begin{array}{l}\text { Approved prostanoids (IV/ } \\
\text { inhaled/SC treprostinil, IV } \\
\text { epoprostenol and inhaled } \\
\text { iloprost oral) ERAs } \\
\text { (ambrisentan, bosentan and } \\
\text { sitaxentan) and PDE-5I (sil- } \\
\text { denafil and tadalafil) }\end{array}$ & $\begin{array}{l}\text { Total mortality and other } \\
\text { clinical endpoints, including } \\
\text { dyspnea, } 6 \mathrm{MWD} \text {, } \\
\text { hemodynamics and AEs }\end{array}$ & $\begin{array}{l}\text { The Jadad score and the } \\
\text { Cochrane Collaboration's } \\
\text { tool }\end{array}$ \\
\hline $\begin{array}{l}\text { Savarese } 2012 \\
{[52]}\end{array}$ & Patients with $\mathrm{PAH}$ & MA & 22 & $\begin{array}{l}\text { Prostanoids (IV } \\
\text { epoprostenol, inhaled } \\
\text { iloprost, oral beraprost and } \\
\text { IV/SC treprostinil), oral ERAs } \\
\text { (bosentan, ambrisentan and } \\
\text { sitaxentan), oral PDE-5Is (sil- } \\
\text { denafil, tadalafil and varde- } \\
\text { nafild) and other drugs (oral } \\
\text { imatinib, aspirin; ROA } \\
\text { unclear) }\end{array}$ & $\begin{array}{l}\text { Primary endpoint: } 6 \mathrm{MWD} \\
\text { Secondary endpoints: all- } \\
\text { cause mortality, } \\
\text { hospitalization for PAH and/ } \\
\text { or lung or heart-lung trans- } \\
\text { plantation, initiation of PAH } \\
\text { rescue therapy }\end{array}$ & Detsky method \\
\hline $\begin{array}{l}\text { Savarese } 2013 \\
{[53]}\end{array}$ & Patients with $\mathrm{PAH}$ & MA & 16 & $\begin{array}{l}\text { Oral PDE-5I (sildenafil and } \\
\text { vardenafil }^{d} \text { ), prostanoids (SC } \\
\text { treprostinil, IV epoprostenol } \\
\text { and inhaled iloprost), oral } \\
\text { ERAs (sitaxentan and } \\
\text { bosentan), oral imatinib }\end{array}$ & $\begin{array}{l}\text { Hemodynamic parameters } \\
\text { (PAP, PVR, RAP and cardiac } \\
\text { index), and clinical events } \\
\text { (all-cause mortality, } \\
\text { hospitalization for PAH and/ } \\
\text { or lung or heart-lung trans- } \\
\text { plantation, initiation of PAH }\end{array}$ & Detsky method \\
\hline
\end{tabular}


Table 2 Characteristics of evidence synthesis studies (Continued)

\begin{tabular}{|c|c|c|c|c|c|c|}
\hline $\begin{array}{l}\text { Study ID } \\
\text { (author year) }\end{array}$ & Patient population & $\begin{array}{l}\text { Type of evidence } \\
\text { synthesis }\end{array}$ & $\begin{array}{l}\text { Number of } \\
\text { studies } \\
\text { included }\end{array}$ & Treatments included & Outcomes included & $\begin{array}{l}\text { Quality assessment tool } \\
\text { (used for included trials) }\end{array}$ \\
\hline & & & & & rescue therapy) & \\
\hline Silva 2017 [54] & $\begin{array}{l}\text { Patients with idiopathic } \\
\text { PAH and associated or } \\
\text { secondary etiologies } \\
\text { (heart failure, CTD- } \\
\text { associated, anorexigen } \\
\text { use, sickle-cell disease, } \\
\text { and HIV) }\end{array}$ & NMA & 20 & $\begin{array}{l}\text { Prostanoids (IV } \\
\text { epoprostenol, SC/oral } \\
\text { treprostinil, oral beraprost } \\
\text { and inhaled iloprost), oral } \\
\text { ERAs (ambrisentan, } \\
\text { bosentan and macitentan), } \\
\text { oral PDE-5Is (sildenafil, tada- } \\
\text { lafil and vardenafil }^{\mathrm{d}} \text { ), sGCS } \\
\text { (oral riociguat) }^{\text {a }}\end{array}$ & $\begin{array}{l}6 \mathrm{MWD}, \text { Cardiac index, PAP, } \\
\text { PVR, clinical worsening, and } \\
\text { mortality }\end{array}$ & $\begin{array}{l}\text { Oxford quality scoring } \\
\text { system }\end{array}$ \\
\hline $\begin{array}{l}\text { Steele } 2010 \\
{[55]}\end{array}$ & $\begin{array}{l}\text { Patients with idiopathic } \\
\text { PAH, or PAH associated } \\
\text { with CTD, CHD or HIV }\end{array}$ & MA & 10 & $\begin{array}{l}\text { Oral bosentan, oral } \\
\text { sitaxentan, inhaled iloprost, } \\
\text { IV epoprostenol, sildenafil, } \\
\text { oral ambrisentan, oral } \\
\text { beraprost, inhaled/SC } \\
\text { treprostinil, oral tadalafil } \\
\text { and oral vardenafil }\end{array}$ & $\begin{array}{l}\text { Primary outcomes: } 6 \mathrm{MWD} \\
\text { Functional Class Secondary } \\
\text { outcomes: mortality, AEs }\end{array}$ & Not reported \\
\hline $\begin{array}{l}\text { Thom } 2015 \\
{[56]}\end{array}$ & Patients with PAH & NMA & $\begin{array}{l}16 \text { (10 RCTs, } 6 \\
\text { observational } \\
\text { studies) }\end{array}$ & $\begin{array}{l}\text { Imatinib (oral) as add-on } \\
\text { therapy to ERA (oral bosen- } \\
\text { tan), oral PDE-5Is (sildenafil } \\
\text { and tadalafil) or prostanoids } \\
\text { (IV epoprostenol, inhaled } \\
\text { iloprost, inhaled/SC trepros- } \\
\text { tinil and oral beraprost) }\end{array}$ & $6 \mathrm{MWD}$ & NICE checklist for RCTs \\
\hline $\begin{array}{l}\text { Tran } 2015[57] \\
\text { [CADTH } \\
\text { report] }\end{array}$ & Patients with PAH & NMA and MA & 20 & $\begin{array}{l}\text { Prostanoids (IV } \\
\text { epoprostenol and SC/IV } \\
\text { treprostinil), oral ERAs } \\
\text { (bosentan, ambrisentan and } \\
\text { macitentan), and oral PDE- } \\
\text { 5Is (sildenafil and tadalafil), } \\
\text { sGCS (oral riociguat) }\end{array}$ & $\begin{array}{l}\text { Clinical outcomes: mortality } \\
\text { (all-cause, PAH-related), } \\
\text { hospitalization, clinical } \\
\text { worsening, NYHAMHO } \\
\text { heart failure Functional } \\
\text { Class, 6MWD, and BDI and } \\
\text { hemodynamic parameters } \\
\text { (PVR, PAP, and cardiac } \\
\text { index) } \\
\text { HRQoL } \\
\text { Safety outcomes: AEs, SAEs } \\
\text { and treatment } \\
\text { discontinuation due to AEs. }\end{array}$ & $\begin{array}{l}\text { A standardized table based } \\
\text { on major items from the } \\
\text { SIGN } 50 \text { instrument. Further } \\
\text { critical appraisal performed } \\
\text { based on input from } \\
\text { clinical experts. }\end{array}$ \\
\hline Vizza 2018 [58] & Patients with $\mathrm{PAH}$ & MA & 6 & $\begin{array}{l}\text { Oral bosentan, oral } \\
\text { ambrisentan, oral riociguat, } \\
\text { oral tadalafil and oral/ } \\
\text { inhaled treprostinil }\end{array}$ & $6 \mathrm{MWD}$ & Not reported \\
\hline $\begin{array}{l}\text { Wang } 2018^{e} \\
{[59]}\end{array}$ & Patients with $\mathrm{PAH}$ & NMA & 45 & $\begin{array}{l}\text { Oral ERAs (ambrisentan, } \\
\text { bosentan, macitentan, } \\
\text { sitaxsentan), oral PDE5Is } \\
\text { (sildenafil, tadalafil, } \\
\text { vardenafil }^{\text {d), }} \text {, prostanoids (IV } \\
\text { epoprostenol, oral/IV/ } \\
\text { inhaled/SC treprostinil, } \\
\text { inhaled iloprost, oral } \\
\text { beraprost), sGCSs (oral } \\
\text { riociguat), sPRA (oral } \\
\text { selexipag) }\end{array}$ & $\begin{array}{l}\text { 6MWD, WHO FC, BDI, } \\
\text { cardiac index, PAP, RAP, } \\
\text { PVR, clinical worsening, } \\
\text { hospitalization, death, SAEs, } \\
\text { and withdrawal }\end{array}$ & Not reported \\
\hline Wei 2016 [60] & $\begin{array}{l}\text { Patients with different } \\
\text { diseases including PAH }\end{array}$ & MA & 24 & $\begin{array}{l}\text { Oral ERAs (bosentan, } \\
\text { ambrisentan and } \\
\text { macitentan); EU authorised }\end{array}$ & AEs & $\begin{array}{l}\text { Cochrane's risk of bias and } \\
\text { GRADE for evidence }\end{array}$ \\
\hline Xing 2011 [61] & $\begin{array}{l}\text { Patients with PAH } \\
\text { (including idiopathic PAH, } \\
\text { familial PAH, as well as } \\
\text { CTD-associated PAH, pul- } \\
\text { monary shut, portal hyper- } \\
\text { tension, HIV infection and } \\
\text { thyroid disease) }\end{array}$ & MA & 10 & $\begin{array}{l}\text { Prostanoids (IV } \\
\text { epoprostenol, IV/SC } \\
\text { treprostinil, oral beraprost } \\
\text { and inhaled iloprost) }\end{array}$ & $\begin{array}{l}6 \mathrm{MWD}, \mathrm{BDI}, \text { cardiac index, } \\
\text { mean PAP, PVR, mortality, } \\
\text { clinical worsening and AEs }\end{array}$ & Jadad scores \\
\hline $\begin{array}{l}\text { Zhang } 2015 \\
\text { [62] }\end{array}$ & Patients with $\mathrm{PAH}$ & MA & 21 & $\begin{array}{l}\text { Oral treatments } \\
\text { (ambrisentan, bosentan, } \\
\text { macitentan, sitaxentan, } \\
\text { sildenafil, tadalafil, riociguat, }_{\text {beraprost, epoprostenol, }} \\
\text { treprostinil, terbogrel }^{\text {d }} \text { and } \\
\text { imatinib }^{\text {d) }}\end{array}$ & $\begin{array}{l}\text { CCW or at least all-cause } \\
\text { mortality }\end{array}$ & Cochrane's risk of bias \\
\hline
\end{tabular}


Table 2 Characteristics of evidence synthesis studies (Continued)

\begin{tabular}{|c|c|c|c|c|c|c|}
\hline $\begin{array}{l}\text { Study ID } \\
\text { (author year) }\end{array}$ & Patient population & $\begin{array}{l}\text { Type of evidence } \\
\text { synthesis }\end{array}$ & $\begin{array}{l}\text { Number of } \\
\text { studies } \\
\text { included }\end{array}$ & Treatments included & Outcomes included & $\begin{array}{l}\text { Quality assessment tool } \\
\text { (used for included trials) }\end{array}$ \\
\hline $\begin{array}{l}\text { Zhang } 2016 \\
\text { [63] }\end{array}$ & Patients with PAH & NMA & 14 & $\begin{array}{l}\text { Prostanoids (IV } \\
\text { epoprostenol, inhaled/IV/ } \\
\text { oral/SC treprostinil, oral } \\
\text { beraprost and inhaled } \\
\text { iloprost) }\end{array}$ & $\begin{array}{l}\text { 6MWD, mortality, } \\
\text { Functional Class, and } \\
\text { discontinuation }\end{array}$ & Not reported \\
\hline $\begin{array}{l}\text { Zhang } 2019 \\
\text { [64] }\end{array}$ & Patients with PAH & NMA & 10 & $\begin{array}{l}\text { Oral ERAs (bosentan, } \\
\text { ambrisentan, macitentan) }\end{array}$ & $\begin{array}{l}\text { Safety outcomes: abnormal } \\
\text { liver function, peripheral } \\
\text { edema and anemia }\end{array}$ & Cochrane's risk of bias \\
\hline $\begin{array}{l}\text { Zheng } 2014 a^{c} \\
{[65]}\end{array}$ & Patients with PAH & MA & 18 & $\begin{array}{l}\text { Oral targeted therapies: } \\
\text { prostanoids (beraprost and } \\
\text { treprostinil), ERAs } \\
\text { (bosentan, ambrisentan and } \\
\text { macitentan), PDE-5Is (sil- } \\
\text { denafil, tadalafil and varde- }^{\text {nafil }}{ }^{\text {) }} \text {, and sGCS (riociguat) }\end{array}$ & $\begin{array}{l}\text { Primary efficacy outcome: } \\
\text { all-cause mortality Second- } \\
\text { ary efficacy outcomes: clin- } \\
\text { ical worsening, WHO } \\
\text { Functional Class, } 6 \mathrm{MWD} \\
\text { Safety outcome: withdrawal } \\
\text { due to AEs }\end{array}$ & Jadad scores \\
\hline $\begin{array}{l}\text { Zheng 2014b } \\
{[66]}\end{array}$ & Patients with PAH & MA & 14 & $\begin{array}{l}\text { Prostanoids (IV } \\
\text { epoprostenol, inhaled, } \\
\text { inhaled/IV/oral/SC } \\
\text { treprostinil, inhaled and oral } \\
\text { beraprost) }\end{array}$ & $\begin{array}{l}\text { Primary efficacy outcome: } \\
\text { all-cause mortality Second- } \\
\text { ary efficacy outcomes: clin- } \\
\text { ical worsening, } 6 \mathrm{MWD} \text {, and } \\
\text { hemodynamic parameters, } \\
\text { including PAP, PVR, cardiac } \\
\text { index, and mixed venous } \\
\text { oxygen saturation. } \\
\text { Safety outcome: withdrawal } \\
\text { due to AEs }\end{array}$ & Jadad scores \\
\hline $\begin{array}{l}\text { Zheng } 2018 \\
\text { [67] }\end{array}$ & Patients with PAH & MA & 25 & $\begin{array}{l}\text { Oral prostanoids } \\
\text { (treprostinil, beraprost), oral } \\
\text { ERAs (ambrisentan, } \\
\text { bosentan, macitentan), oral } \\
\text { PDE-5Is (sildenafil, tadalafil, } \\
\text { vardenafil }^{d} \text { ), sGCSs (oral rio- } \\
\text { ciguat), sPRA (oral }_{\text {selexipag) }}\end{array}$ & $\begin{array}{l}\text { Primary outcome: } \\
\text { composite clinical } \\
\text { worsening } \\
\text { Secondary outcomes: all- } \\
\text { cause mortality, lung trans- } \\
\text { plantation, admission to } \\
\text { hospital, treatment escal- } \\
\text { ation, WHO FC improve- } \\
\text { ment, symptomatic } \\
\text { progression and 6MWD }\end{array}$ & Jadad score \\
\hline Zhu 2012 [68] & Patients with PAH & MA & 7 & $\begin{array}{l}\text { Oral PDE-5Is (sildenafil and } \\
\text { tadalafil) oral ERAs (bosen- } \\
\text { tan, sitaxentan and ambri- } \\
\text { sentan), prostanoids (IV } \\
\text { epoprostenol, inhaled ilo- } \\
\text { prost and IV treprostinil) }\end{array}$ & $\begin{array}{l}6 \mathrm{MWD} \text {, clinical worsening, } \\
\text { mortality (data not shown) }\end{array}$ & $\begin{array}{l}\text { Moher } 1998 \text { reference } \\
\text { provided for the quality } \\
\text { assessment }\end{array}$ \\
\hline
\end{tabular}

AEs Adverse events, BDI Borg dyspnea index, CCW Combined clinical worsening, CHD Congenital heart disease, CTD Connective tissue disease, ERAs Endothelin Receptor Antagonists, FC Functional class, FPAH Familial PAH, HRQOL Health related quality of life, IPAH Idiopathic PAH, MA Meta-analysis, NMA Network metaanalysis, NT-proBNP N-terminal probrain natriuretic peptide, NYHA/ WHO New York Heart Association/World Health Organization, IV Intravenous, PAH Pulmonary arterial hypertension, PAP Pulmonary arterial pressure, PCAs Prostacyclin analogues, PDE-5/s Phosphodiesterase 5 Inhibitors, PH Pulmonary hypertension, PVR Pulmonary vascular resistance, RAP Right atrial pressure, SAEs Severe adverse events, sGCSs Soluble guanylate cyclase stimulators, sPRAs Selective non-prostanoid prostacyclin receptor agonists, SC subcutaneous, SSC-PAH Pulmonary arterial hypertension related to systemic sclerosis, 6 MWD Six minute walking distance ${ }^{a}$ Although Badiani 2015 reported that prostanoids with IV/inhaled/SC ROA were considered for evaluation, trials on prostanoids with these ROAs were not included in the analysis. No justification provided. In Fox 2011, sitaxsentan, ambrisentan and vardenafil were included in the search strategy of the review, however, trials with these therapies were not included in the analysis. No justification provided. ${ }^{~}$ In Zheng 2014a, trials on sitaxentan were excluded from the analysis as it was withdrawn from the market due to liver toxicity. The trial on selexipag was also excluded but provided no justification for the exclusion. ${ }^{\mathrm{d}}$ Treatments that have not been approved or made to any markets for adult patients with PAH. ${ }^{\text {In }}$ Wang 2018, a subgroup analysis excluding sitaxsentan was conducted for network comparison of drugs in use on the market

example, claims of PDE-5I monotherapy being superior and a therapy of choice based on older, short-term trials (e.g. Singh 2006 [70], Galie 2005a [71]) are not aligned with evidence from more recent, longer-term studies suggesting that PDE-5I monotherapy is inferior to combination therapy (e.g. SERAPHIN [72], AMBITION [73], GRIPHON [74]). Such inconsistencies across studies challenge a robust interpretation of results for decision makers concerned with a comprehensive assessment of all approved treatments, given the dearth of direct comparisons in RCTs.

\section{Credibility}

Of the 52 studies reviewed, six were scored as strong in terms of credibility, 18 as neutral, and the remaining 28 as weak.

The majority of studies attempted to identify all relevant RCTs. Some studies did not search all of the most relevant databases, i.e. MEDLINE, Embase, CENTRAL $[18,29,32,34,35,44,45,52,53,68]$. Several studies did not provide details of the search strategy [18-21, 24-26, $29,31,32,35,36,39,40,44-46,49,50,52-55,59-61$, $63,67,68]$ and one study did not provide any details on the search strategy and searched databases [58]. 

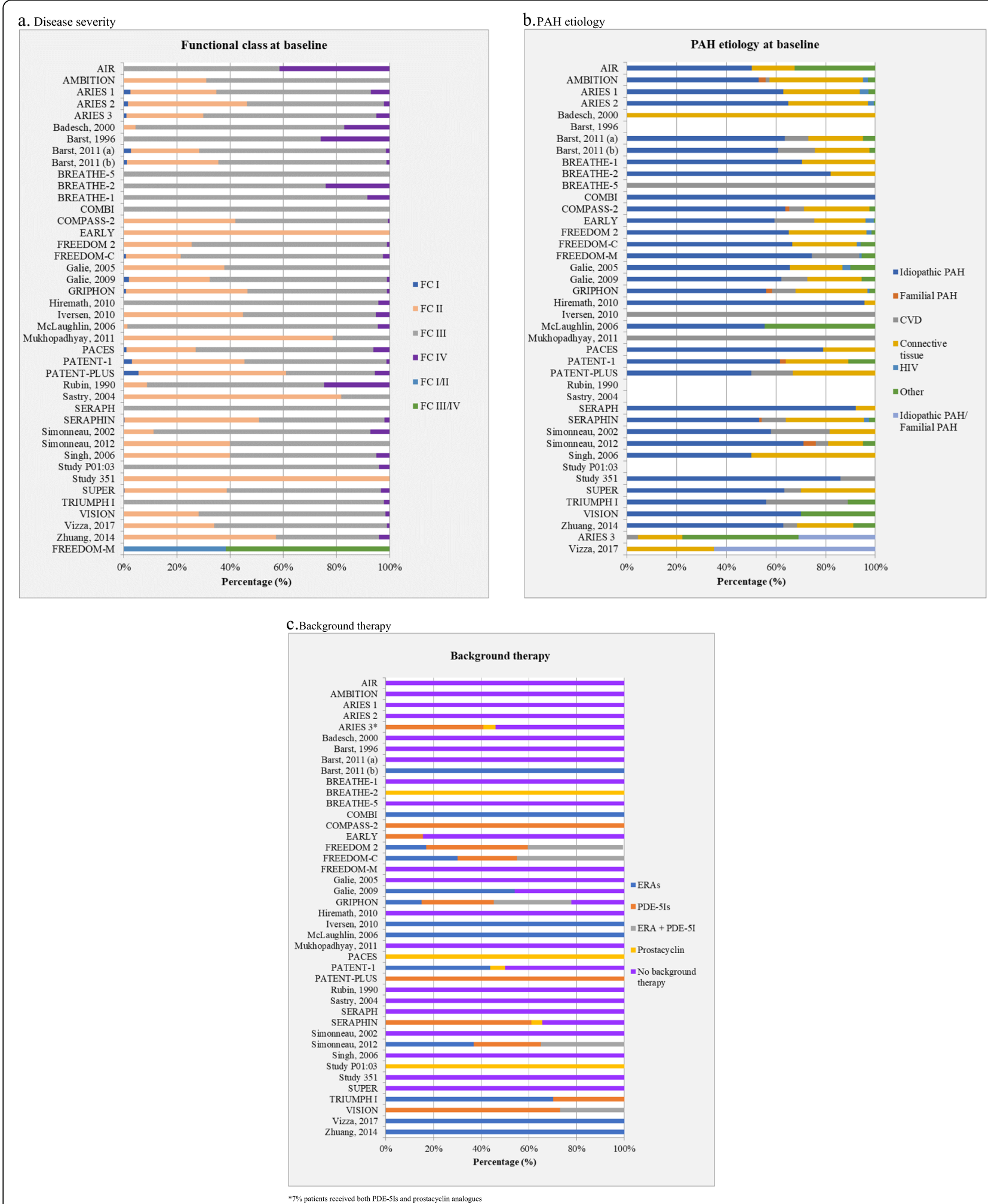

Fig. 1 a-c Disease severity, PAH etiology and background therapy across included RCTs 


\begin{tabular}{|c|c|c|c|c|c|c|}
\hline Study & Relevance & Credibility & Analysis & $\begin{array}{l}\text { Reporting Quality \& } \\
\text { Transparency }\end{array}$ & Interpretation & Conflict of Interest \\
\hline Avouac 2008 & Weakness & Weakness & Weakness & Weakness & Strength & Weakness \\
\hline Badiani 2016 & Weakness & Weakness & Weakness & Weakness & Neutral & Strength \\
\hline Bai 2011 & Strength & Neutral & Weakness & Weakness & Neutral & Strength \\
\hline Barnes 2019 & Strength & Neutral & Neutral & Neutral & Neutral & Strength \\
\hline Biondi-Zoccia 2013 & Weakness & Weakness & Weakness & Neutral & Weakness & Strength \\
\hline Chen 2009 & Neutral & Strength & Neutral & Neutral & Strength & Strength \\
\hline Coeyteaux 2014 & Neutral & Weakness & Weakness & Weakness & Neutral & Strength \\
\hline Dranitsaris 2009 & Neutral & Weakness & Neutral & Weakness & Neutral & Weakness \\
\hline Duo-Ji 2017 & Neutral & Neutral & Weakness & Weakness & Weakness & Strength \\
\hline Fox 2011 & Neutral & Neutral & Neutral & Neutral & Strength & Weakness \\
\hline Fox 2016 & Strength & Neutral & Weakness & Weakness & Neutral & Weakness \\
\hline Gabler 2012 & Strength & Strength & Neutral & Neutral & Strength & Weakness \\
\hline Galie 2009b & Neutral & Weakness & Neutral & Weakness & Neutral & Weakness \\
\hline Gao 2017 & Weakness & Weakness & Weakness & Weakness & Neutral & Strength \\
\hline He 2010 & Weakness & Weakness & Weakness & Weakness & Weakness & Strength \\
\hline Igarashi 2016 & Weakness & Weakness & Neutral & Weakness & Neutral & Weakness \\
\hline Jain 2017 & Neutral & Neutral & Neutral & Strength & Weakness & Neutral \\
\hline Khouri 2018 & Weakness & Weakness & Weakness & Weakness & Weakness & Neutral \\
\hline Kirtania 2019 & Neutral & Neutral & Neutral & Neutral & Neutral & Strength \\
\hline Kuwana 2013 & Weakness & Neutral & Weakness & Neutral & Neutral & Neutral \\
\hline Lajoie 2016 & Strength & Strength & Neutral & Strength & Neutral & Neutral \\
\hline Lajoie 2018 & Neutral & Neutral & Weakness & Weakness & Weakness & Neutral \\
\hline Lei 2020 & Weakness & Weakness & Weakness & Weakness & Weakness & Neutral \\
\hline Li 2013 & Neutral & Neutral & Neutral & Strength & Neutral & Strength \\
\hline Li 2019 & Neutral & Weakness & Neutral & Neutral & Weakness & Strength \\
\hline Lin 2018 & Strength & Neutral & Weakness & Strength & Strength & Strength \\
\hline Liu 2016 & Weakness & Weakness & Weakness & Weakness & Strength & Strength \\
\hline Macchia 2007 & Neutral & Weakness & Neutral & Strength & Strength & Weakness \\
\hline Macchia 2010 & Neutral & Weakness & Neutral & Neutral & Strength & Strength \\
\hline Pan 2017 & Weakness & Weakness & Weakness & Weakness & Neutral & Strength \\
\hline Pan 2018 & Weakness & Weakness & Neutral & Neutral & Strength & Weakness \\
\hline Paramothayan 2009 & Strength & Neutral & Strength & Neutral & Strength & Strength \\
\hline Petrovic 2020a & Neutral & Weakness & Weakness & Neutral & Neutral & Neutral \\
\hline Petrovic 2020b & Neutral & Neutral & Strength & Neutral & Strength & Neutral \\
\hline Ryerson 2010 & Neutral & Strength & Neutral & Weakness & Strength & Neutral \\
\hline Savarese 2012 & Strength & Neutral & Neutral & Weakness & Neutral & Weakness \\
\hline Savarese 2013 & Weakness & Weakness & Neutral & Strength & Neutral & Weakness \\
\hline Silva 2017 & Weakness & Neutral & Weakness & Neutral & Weakness & Weakness \\
\hline Steele 2010 & Neutral & Weakness & Neutral & Neutral & Strength & Neutral \\
\hline Thom 2015 & Neutral & Strength & Strength & Neutral & Strength & Neutral \\
\hline Trans 2015 & Neutral & Strength & Strength & Strength & Strength & Neutral \\
\hline Vizza 2018 & Weakness & Weakness & Weakness & Weakness & Weakness & Weakness \\
\hline Wang 2018 & Neutral & Weakness & Weakness & Neutral & Weakness & Neutral \\
\hline Wei 2016 & Weakness & Weakness & Weakness & Neutral & Weakness & Strength \\
\hline Xing 2011 & Neutral & Weakness & Weakness & Neutral & Neutral & Strength \\
\hline Zhang 2015 & Neutral & Neutral & Strength & Neutral & Neutral & Strength \\
\hline Zhang 2016 & Weakness & Weakness & Weakness & Weakness & Weakness & Weakness \\
\hline Zhang 2019 & Neutral & Weakness & Weakness & Neutral & Neutral & Neutral \\
\hline Zheng 2014a & Neutral & Neutral & Weakness & Weakness & Weakness & Neutral \\
\hline Zheng 2014b & Neutral & Neutral & Weakness & Weakness & Neutral & Strength \\
\hline Zheng 2018 & Neutral & Weakness & Neutral & Neutral & Neutral & Neutral \\
\hline Zhu 2012 & Weakness & weakness & eness & & Neutral & Strengt \\
\hline
\end{tabular}

Fig. 2 Overview of quality assessment

Table 3 Number of studies with judgement in each domain

\begin{tabular}{llll}
\hline Domains of Quality Appraisal & Strength & Neutral & Weakness \\
\hline Relevance & 8 & 26 & 18 \\
Credibility & 6 & 18 & 28 \\
Analysis & 5 & 20 & 27 \\
Reporting Quality \& Transparency & 7 & 22 & 23 \\
Interpretation & 15 & 23 & 14 \\
Conflict of Interest & 22 & 16 & 14 \\
\hline
\end{tabular}

The proposed methodology was found to be relevant to answer the decision problem in almost all included studies. Some studies did not conduct a quality assessment of included RCTs [18, 24, 28, 44, 55, 58, 59]. Several studies did not provide the results of the RCT quality assessment or discuss implications for the analysis in case of poor quality RCTs $[21,23,30,31,36,39$, 46, 62, 63, 68].

Given the absence of randomization across the RCTs included in an MA or NMA, the assessment of effect modifiers is essential to validate assumptions around homogeneity, consistency and transitivity [75, 76]. Effect modifiers are study and patient characteristics associated with treatment effects, capable of modifying (positively or negatively) the observed effect of a risk factor on 
disease status. Potential effect modifiers in PAH include patient baseline characteristics such as 6MWD, WHO functional class, disease duration, background therapies and etiology; and study design characteristics such as study duration and imputation rules. As the overview of design and patient baseline characteristics of included PAH RCTs (see Fig. 1a-c; Figure S3a-d in the electronic supplementary material) demonstrates, substantial between-study heterogeneity is a feature of every evidence synthesis study in PAH. The majority of studies did not offer a comprehensive assessment prior to analysis or identify imbalances in effect modifiers across the RCTs $[17,18,20,21,23-27,30,32,34,39,44-47,49$, $51,53,54,58-66,68,69]$.

\section{Analysis}

Of the 52 studies reviewed, five were scored as strong in terms of analysis, 20 as neutral, and the remaining 27 as weak.

Preservation of study randomization of included RCTs was fulfilled by almost all included studies except in five studies with single-arm $[36,39,56]$, retrospective comparative [35] or open-label extension design [58]. Several MAs adopted an approach whereby, for multi-arm trials, the control group was split and the sample size halved [34, 37, 60, 67]. Though outlined in the Cochrane Handbook for Systematic Reviews of Interventions [12], this approach effectively breaks randomization and should therefore be avoided. Other forms of evidence synthesis (e.g. NMA) are more appropriate in this case. Of the included NMA studies with closed loops, most assessed the consistency between the direct and indirect evidence $[13,14,50,59,64]$.

Common types of analysis to address imbalance in the distribution of treatment effect modifiers include subgroup and sensitivity analysis, meta-regression and using individual patient data. Only about a third of included studies attempted to address between-study heterogeneity $[22,24,33,35,37,38,40,44,48,50-53,56,57,61]$. The majority of included studies (primarily MAs) used a fixed effects model unless marked heterogeneity was detected (typically assessed using the Cochran Q-test or $I^{2}$ statistic), in which case a random effects model was used $[17,20,25,29,31,34,39,44,45,48,51,59,60,62,65-$ 67]. Some studies only fitted a random effects model $[19,20,23,26,27,35,40,46,47,49,50,64]$, whereas others only fitted a fixed effects model $[28,30,38]$. The deviance information criterion commonly formed the sole criterion for assessing model fit in the included NMA studies [18, 21, 32] except for Tran et al. 2015 [57], Petrovic 2020a [48] and Petrovic 2020b [49] who assessed model fit based on deviance information criterion and a comparison of the residual deviance with the number of unconstrained data points.
Lastly, several studies pooled treatments at the class level, usually without sound justification for the assumption of a class effect. Very few studies refrained from lumping treatments, doses and co-treatments together $[28,48,50,55-57,62,64]$.

\section{Reporting quality \& transparency}

Of the 52 studies reviewed, seven were scored as strong in terms of their reporting quality and transparency, 22 as neutral, and the remaining 23 as weak.

All included NMA studies presented a network diagram, except Zhang et al. 2016 [63]. Two of the 11 included NMA studies did not present details of the number and/or RCTs per pairwise comparison [18, 30]. Separate reporting of direct and indirect comparisons was omitted in six NMA studies [18, 25, 30, 49, 50, 56]. A ranking of interventions according to the reported treatment effects was provided by two-third of the included NMA studies $[18,25,33,42,49,50,57,59,63$, 64], some of which did not report associated uncertainty measures. The reporting of all pairwise contrasts between interventions, along with measures of uncertainty, was not adhered by two of the 11 NMA studies $[18,56]$.

The reporting of individual study results was omitted or not fully reported by 14 of the 52 studies [21, 25, 30, $32,38,42,46,49,50,55,57,59,63,64]$. Overall, 37 of the included studies either completely omitted a discussion or provided a very brief reference to heterogeneity across studies without a specific discussion of the potential impact of differences in patient characteristics on observed results $[17-21,23-27,29-32,34-36,38,39$, $46,47,49,51,52,54,58-65,67,68]$.

\section{Interpretation}

Overall, 15 of the 52 studies reviewed were scored as strong in terms of their interpretation of study findings, 23 as neutral, and the remaining 14 as weak.

A number of studies were scored as 'weak' when authors did not contextualize results considering limitations [31, 34, 38, 39, 58, 63], or endorsed specific treatments over others without any discussion of between-study heterogeneity and/or despite pooling of active therapies $[20,21,25,33,39,59,60]$. For example, Jain et al. 2017 [33] combined trials [74, 77, 78] in their primary analysis that differed in patients' severity level and provision of background therapies.

\section{Conflict of interest}

Among included studies, 22 were scored as strong in terms of conflict of interest, 16 as neutral, and the remaining 14 as weak.

Less than a third of all assessed studies provided either no information about conflicts of interest or insufficiently detailed author disclosures. Other studies 
reported no personal or financial relationships, or clearly stated author contributions in case of personal or final relationships of affiliations that could have biased the respective study.

\section{Discussion}

The objective of this study was to systematically appraise all identified MA/NMA studies in PAH and assess their quality given that such studies are taken into consideration for evidence-based decision-making. To our knowledge, this is the first study of this type in PAH. Overall, the appraisal found most evidence synthesis studies to be of low quality.

Most included evidence syntheses were found not to have defined the decision problem (i.e. the research question underpinning a study), population, selection of comparisons and outcome selection that is compatible or aligned with current clinical practice and treatment guidelines $[2,79]$. Of note, the majority of the studies $[18-26,29,30,32,34,36,40,44-49,51,54,55,57-60$, 62-66, 68] included trials that do not reflect today's clinical practice. For example, the BREATHE-2 [80] and PACES [81] trials investigated bosentan and sildenafil, respectively, as add-on therapy to IV epoprostenol. By contrast, PAH management today typically involves treatment initiation of oral therapy with an ERA and/or PDE-5I in low or intermediate-risk patients comprising the vast majority of patients, whereas parenteral prostacyclins would only be considered or added for high-risk patients [6].

Notably, clinical trial design has evolved from a preponderance of small, short-term and often open-label studies in treatment-naive patients with severe PAH to larger, longer-term and event-driven trials (such as COMPASS-2 [82], SERAPHIN [72], AMBITION [73], GRIPHON [74]) in largely treatment-experienced and less severe patient populations. Similarly, primary endpoint definition has gradually shifted from improvement in 6MWD to morbidity and mortality as a composite endpoint (with components such as all-cause death, PAH-related hospitalization or disease worsening) which is considered to be a more patient- and clinically relevant endpoint [83-85].

While these changes in trial design and PAH management pose challenges for studies synthesizing evidence generated across such large time spans, a transparent interpretation of findings in recent MA/NMA studies in relation to present clinical practice and guidance was found to be lacking.

A related shortcoming of appraised studies is the choice of outcomes analyzed, which was found to be selective, incomprehensive, and usually not accompanied by clear justification. The most commonly assessed outcome was 6MWD - despite failure of multiple studies to consistently establish significant associations between 6MWD and clinically more relevant outcomes such PAH-related hospitalization, lung transplantation, initiation of rescue therapy or death [28, 29, 44, 52, 86, 87]. Moreover, the assessed evidence synthesis studies generally neither presented a review of the outcome definitions and outcome measures of included trials, nor an assessment of imputation rules for handling missing data.

Mortality was less commonly assessed, which reflects the inherent challenges in designing clinical trials of PAH therapies to detect statistically significant or clinically meaningful differences in mortality. Replication of earlier trials (e.g. Barst 1996 [78]) showing survival benefit over a very short time period and placebo-controlled RCTs comparing monotherapy with no therapy in treatment-naïve patients would be considered unethical today.

Another crucial drawback in most included studies is the lack of a thorough assessment of key effect modifiers prior to the analysis. As the graphs presenting patient baseline characteristics across $\mathrm{PAH}$ trials demonstrate (see Fig. 1a-c; Figure S3a-d in the electronic supplementary material), there is marked between-study heterogeneity. One recurring observation was that most evidence synthesis studies included a mix of $\mathrm{PAH}$ and non-PAH patients populations, as in the aerosolized iloprost randomized (AIR) study [88] which included PAH and chronic thromboembolic pulmonary hypertension (CTEPH) patients.

Only a handful of studies sought to address such potential systematic differences in the effect modifiers through means of subgroup/sensitivity analyses, metaregression. This may be due to limited subgroup data available from published PAH RCTs, and challenges around smaller sample sizes associated with subgroup data which results in wider uncertainty estimates and lower likelihood of detecting significant relative treatment effects.

In terms of results synthesis, several studies were found to pool treatments at the drug class level. Best practices guidelines in evidence synthesis, such as NICE DSU TSD 7 [13], recommend against pooling treatment doses or treatments into drug classes since characteristics of the underlying trial population or efficacy/safety trial results may be different.

This review has some limitations. A thorough assessment of the quality of MA/NMA studies is limited by the heterogeneity across included trials. A detailed assessment of between-study heterogeneity in each included MA/NMA was beyond the scope of the review. Nevertheless, a preliminary assessment of patients' baseline characteristics of all PAH trials included across the appraised MA/NMA studies was considered reflective of 
most studies. Results or analyses relating to PAH subgroups by etiology, severity or age were not explored further due to no or very limited studies focusing on these specific sub-populations.

\section{Conclusion}

This is the first critical appraisal of published MA/NMA studies in PAH, suggesting overall low quality and validity of efforts synthesizing PAH evidence. As our study demonstrates, this has important implications for clinical decision-making and future research. First, the choice of optimal therapy to maximize patient outcomes should also be guided by a consideration of the limitations of published MA/NMA studies highlighted in this study. Second, future attempts of evidence synthesis in PAH should improve the level of validity and scrutiny to meaningfully address challenges arising from an evolving therapeutic landscape. This should include the definition of decision problems that are aligned with today's clinical practice and treatment guidelines, justification of key analysis assumptions, a comprehensive interrogation of the evidence base prior to analysis, use of individual patient data to mitigate issues of heterogeneity, and a transparent presentation of results and associated uncertainty measures for all relevant outcomes.

\section{Supplementary information}

Supplementary information accompanies this paper at https://doi.org/10. 1186/s12890-020-01241-4.

Additional file 1: Table S1. Eligibility criteria of the Systematic Literature Review. Table S2a-d. Search strategies (September 2018). Table S2e-h. Search strategies (April 2020 update). Table S3. Quality assessment of included evidence synthesis studies. Figure S1. Treatment algorithm. Figure S2a. PRISMA diagram showing study selection process (September 2018). Figure S2b. PRISMA diagram showing study selection process (April 2020 update). Figure S3a-d. Mean age, gender, disease duration and 6MWD in included RCTs.

\section{Abbreviations \\ CTEPH: Chronic thromboembolic pulmonary hypertension; ERA: Endothelin receptor antagonist; ERS: European Respiratory Society; ESC: The European Society of Cardiology; HRQoL: Health-related-quality-of -life; ISPOR: International Society for Pharmacoeconomics and Outcomes Research; IV: Intravenous; MA: Meta-analyses; NICE: The National Institute for Health and Care Excellence; NMA: Network-meta-analyses; PAH: Pulmonary arterial hypertension; PDE-51: Phosphodiesterase-5 inhibitors; \\ PICOS: Participants, interventions, comparisons, outcomes, and study design; PH : Pulmonary Hypertension; PRISMA: Preferred Reporting Items for Systematic Reviews and Meta-Analyses; PVR: Pulmonary vascular resistance; RCTs: Randomized controlled trials; sGCS: Soluble guanylate cyclase stimulator}

\section{Acknowledgements}

The authors thank Dr. Rainer Zimmerman for his review and valuable comments on the manuscript from a clinical perspective.

\section{Authors' contributions}

$A B$ and $E D$ conceptualized the study, while $A B, A K, E D$ and $M S$ designed the study. BG and MS led the study conduct, while all authors jointly analysed and interpreted the study results. BG and MS led the write-up of the manuscript, while $A B, E D$ and $A K$ reviewed the drafted work. All authors approved the submitted version and agreed to be personally accountable for the author's own contributions and to ensure that questions related to the accuracy or integrity of any part of the work, even ones in which the author was not personally involved, are appropriately investigated, resolved, and the resolution documented in the literature.

\section{Funding}

Sponsorship for this study and article processing charges were funded by Actelion Pharmaceuticals Ltd. All authors had full access to all of the data in this study and take complete responsibility for the integrity of the data and accuracy of the data analysis.

\section{Availability of data and materials}

The datasets analyzed during the current study are available from the corresponding author on reasonable request.

\section{Ethics approval and consent to participate}

This article is based on previously conducted studies and does not contain any studies with human participants or animals performed by any of the authors.

\section{Consent for publication}

Not applicable.

\section{Competing interests}

The authors declare that they have no competing interests.

\section{Author details}

${ }^{1}$ IQVIA, 210 Pentonville Road, London N1 9JY, UK. ${ }^{2}$ Actelion Pharmaceuticals Ltd, Gewerbestrasse 16, CH-4123 Allschwil, Switzerland. ${ }^{3}$ IQVIA, Herikerbergweg 314, 1101 CT Amsterdam, Netherlands. ${ }^{4}$ Department of Statistical Science, University College London, London WC1E 6BT, UK.

Received: 19 November 2019 Accepted: 17 July 2020

Published online: 28 July 2020

References

1. Humbert M, Guignabert C, Bonnet S, et al. Pathology and pathobiology of pulmonary hypertension: state of the art and research perspectives. Eur Respir J. 2019;53(1):1801887.

2. Galie N, Humbert M, Vachiery JL, Gibbs S, Lang I, Torbicki A, Simonneau G, Peacock A, Vonk Noordegraaf A, Beghetti M, et al. 2015 ESC/ERS guidelines for the diagnosis and treatment of pulmonary hypertension: the joint task force for the diagnosis and treatment of pulmonary hypertension of the European Society of Cardiology (ESC) and the European Respiratory Society (ERS): endorsed by: Association for European Paediatric and Congenital Cardiology (AEPC), International Society for Heart and Lung Transplantation (ISHLT). Eur Heart J. 2016;37(1):67-119.

3. D'Alonzo GE, Barst RJ, Ayres SM, Bergofsky EH, Brundage BH, Detre KM, Fishman AP, Goldring RM, Groves BM, Kernis JT, et al. Survival in patients with primary pulmonary hypertension. Results from a national prospective registry. Ann Intern Med. 1991;115(5):343-9.

4. Farber HW, Miller DP, Poms AD, Badesch DB, Frost AE, Muros-Le Rouzic E, Romero AJ, Benton WW, Elliott CG, McGoon MD, et al. Five-year outcomes of patients enrolled in the REVEAL registry. Chest. 2015;148(4):1043-54.

5. EMA. EU/3/04/234. 2018. https://www.ema.europa.eu/en/medicines/human/ orphan-designations/eu304234.

6. Galiè N, Channick RN, Frantz RP, et al. Risk stratification and medical therapy of pulmonary arterial hypertension. Eur Respir J. 2019;53(1):1801889.

7. Jansen JP, Fleurence R, Devine B, Itzler R, Barrett A, Hawkins N, Lee K, Boersma C, Annemans L, Cappelleri JC. Interpreting indirect treatment comparisons and network meta-analysis for health-care decision making: report of the ISPOR task force on indirect treatment comparisons good research practices: part 1. Value Health. 2011;14(4):417-28.

8. Higgins JP, Altman DG, Gotzsche PC, Juni P, Moher D, Oxman AD, Savovic J, Schulz KF, Weeks L, Sterne JA. The Cochrane Collaboration's tool for assessing risk of bias in randomised trials. BMJ (Clin Res Ed). 2011;343:d5928.

9. Moher D, Liberati A, Tetzlaff J, Altman DG. Preferred reporting items for systematic reviews and meta-analyses: the PRISMA statement. PLoS Med. 2009;6(7):e1000097. 
10. NICE. Guide to the methods of technology appraisal 2013. National Institute for Health and Care Excellence (NICE). 2013. Available from: https://www. nice.org.uk/process/pmg9/resources/guide-to-the-methods-of-technologyappraisal-2013-pdf-2007975843781.

11. CRD. Systematic reviews: CRD's guidance for undertaking reviews in health care. Centre for Reviews and Dissemination (CRD). 2009. Available from: https://www.york.ac.uk/media/crd/Systematic_Reviews.pdf.

12. Higgins JP, Green S. Cochrane handbook for systematic reviews of interventions version 5.1.0 [updated March 2011] the Cochrane Collaboration. 2011. Available from www.handbook.cochrane.org.

13. Ades AE, Caldwell DM, Reken S, Welton NJ, Sutton AJ, Dias S. NICE decision support unit technical support documents. In: Evidence synthesis of treatment efficacy in decision making: a reviewer's checklist. London: National Institute for Health and Care Excellence (NICE); 2012.

14. Jansen JP, Trikalinos T, Cappelleri JC, Daw J, Andes S, Eldessouki R, Salanti G. Indirect treatment comparison/network meta-analysis study questionnaire to assess relevance and credibility to inform health care decision making: an ISPOR-AMCP-NPC good practice task force report. Value Health. 2014; 17(2):157-73.

15. Hutton B, Salanti G, Caldwell DM, Chaimani A, Schmid CH, Cameron C, loannidis JP, Straus S, Thorlund K, Jansen JP, et al. The PRISMA extension statement for reporting of systematic reviews incorporating network metaanalyses of health care interventions: checklist and explanations. Ann Intern Med. 2015;162(11):777-84.

16. Guyatt GH, Oxman AD, Schunemann HJ, Tugwell P, Knottnerus A. GRADE guidelines: a new series of articles in the journal of clinical epidemiology. J Clin Epidemiol. 2011;64(4):380-2.

17. Avouac J, Wipff J, Kahan A, Allanore Y. Effects of oral treatments on exercise capacity in systemic sclerosis related pulmonary arterial hypertension: a meta-analysis of randomised controlled trials. Ann Rheum Dis. 2008;67(6): 808-14.

18. Badiani B, Messori A. Targeted treatments for pulmonary arterial hypertension: interpreting outcomes by network meta-analysis. Heart Lung Circ. 2016;25(1):46-52.

19. Bai $Y$, Sun $L$, Hu S, Wei $Y$. Combination therapy in pulmonary arterial hypertension: a meta-analysis. Cardiology. 2011;120(3):157-65.

20. Barnes H, Brown Z, Burns A, Williams T. Phosphodiesterase 5 inhibitors for pulmonary hypertension. Cochrane Database Syst Rev. 2019;1:Cd012621.

21. Biondi-Zoccai G, D'Ascenzo F, Cannillo M, Welton NJ, Marra WG, Omede P, Libertucci D, Fusaro E, Capriolo M, Perversi J, et al. Choosing the best first line oral drug agent in patients with pulmonary hypertension: evidence from a network meta-analysis. Int J Cardiol. 2013;168(4):4336-8.

22. Chen YF, Jowett S, Barton P, Malottki K, Hyde C, Gibbs JSR, Pepke-Zaba J, Fry-Smith A, Roberts J, Moore D. Clinical and cost-effectiveness of epoprostenol, iloprost, bosentan, sitaxentan and sildenafil for pulmonary arterial hypertension within their licensed indications: a systematic review and economic evaluation. Health Technol Assess. 2009;13(49):ix-320.

23. Coeytaux RR, Schmit KM, Kraft BD, Kosinski AS, Mingo AM, Vann LM, Gilstrap DL, Hargett CW, Heidenfelder B, Dolor RJ, et al. Comparative effectiveness and safety of drug therapy for pulmonary arterial hypertension. Chest. 2014; 145(5):1055-63.

24. Dranitsaris $G$, Mehta S. Oral therapies for the treatment of pulmonary arterial hypertension: a population-based cost-minimization analysis. Appl Health Econ Health Policy. 2009;7(1):43-59.

25. Duo-Ji MM, Long ZW. Comparative efficacy and acceptability of endothelin receptor antagonists for pulmonary arterial hypertension: a network metaanalysis. Int J Cardiol. 2017;234:90-8.

26. Fox BD, Shimony A, Langleben D. Meta-analysis of monotherapy versus combination therapy for pulmonary arterial hypertension. Am J Cardiol. 2011;108(8):1177-82.

27. Fox BD, Shtraichman O, Langleben D, Shimony A, Kramer MR. Combination therapy for pulmonary arterial hypertension: a systematic review and metaanalysis. Can J Cardiol. 2016:32(12):1520-30.

28. Gabler NB, French B, Strom BL, Palevsky HI, Taichman DB, Kawut SM, Halpern SD. Validation of 6-minute walk distance as a surrogate end point in pulmonary arterial hypertension trials. Circulation. 2012;126(3):349-56.

29. Galie N, Manes A, Negro L, Palazzini M, Bacchi-Reggiani ML, Branzi A. A meta-analysis of randomized controlled trials in pulmonary arterial hypertension. Eur Heart J. 2009b;30(4):394-403.

30. Gao XF, Zhang JJ, Jiang XM, Ge Z, Wang ZM, Li B, Mao WX, Chen SL. Targeted drugs for pulmonary arterial hypertension: a network meta- analysis of 32 randomized clinical trials. Patient Prefer Adherence. 2017;11: 871-85.

31. He B, Zhang F, Li X, Tang C, Lin G, Du J, Jin H. Meta-analysis of randomized controlled trials on treatment of pulmonary arterial hypertension. Circ J. 2010;74(7):1458-64.

32. Igarashi A, Inoue S, Ishii T, Tsutani $K$, Watanabe $H$. Comparative effectiveness of oral medications for pulmonary arterial hypertension: network metaanalysis. Int Heart J. 2016;57(4):466-72.

33. Jain S, Khera R, Girotra S, Badesch D, Wang Z, Murad MH, Blevins A, Schmidt GA, Singh S, Gerke AK. Comparative effectiveness of pharmacologic interventions for pulmonary arterial hypertension: a systematic review and network meta-analysis. Chest. 2017;151(1):90-105.

34. Khouri C, Lepelley M, Roustit M, Montastruc F, Humbert M, Cracowski JL. Comparative safety of drugs targeting the nitric oxide pathway in pulmonary hypertension: a mixed approach combining a meta-analysis of clinical trials and a disproportionality analysis from the World Health Organization pharmacovigilance database. Chest. 2018;154(1):136-47.

35. Kirtania L, Maiti R, Srinivasan A, Mishra A. Effect of combination therapy of endothelin receptor antagonist and phosphodiesterase-5 inhibitor on clinical outcome and pulmonary haemodynamics in patients with pulmonary arterial hypertension: a meta-analysis. Clin Drug Invest. 2019; 39(11):1031-44

36. Kuwana M, Watanabe $H$, Matsuoka N, Sugiyama N. Pulmonary arterial hypertension associated with connective tissue disease: meta-analysis of clinical trials. BMJ Open. 2013;3 (8) (no pagination):e003113.

37. Lajoie AC, Lauziere G, Lega JC, Lacasse Y, Martin S, Simard S, Bonnet S, Provencher S. Combination therapy versus monotherapy for pulmonary arterial hypertension: a meta-analysis. Lancet Respir Med. 2016;4(4):291-305.

38. Lajoie AC, Guay CA, Lega JC, Lauziere G, Simard S, Lambert C, Lacasse Y, Bonnet $\mathrm{S}$, Provencher $\mathrm{S}$. Trial duration and risk reduction in combination therapy trials for pulmonary arterial hypertension: a systematic review. Chest. 2018;153(5):1142-52.

39. Lei $Y$, Zhang $X$, Lin H, Feng Y, Wang J, Luo R. The effects of oral treatment for systemic sclerosis related pulmonary arterial hypertension: a systematic review and meta-analysis. Mod Rheumatol. 2020:1-11.

40. Li T, Chen Y, Zang W, Geng N, Ma S, Li X. Prostacyclin and its analogues in pulmonary artery hypertension: a meta-analysis. Curr Med Res Opin. 2013; 29(8):889-99.

41. Li X, Li T. Combined methods (formal adjusted indirect comparison, metaanalysis and principal component analysis) comparisons of the safety and efficacy of ambrisentan, bosentan, and sildenafil in the patients with pulmonary arterial hypertension. Front Pharmacol. 2020;11:400.

42. Lin H, Wang M, Yu Y, Qin Z, Zhong X, Ma J, Zhao F, Zhang X. Efficacy and tolerability of pharmacological interventions for pulmonary arterial hypertension: a network meta-analysis. Pulm Pharmacol Ther. 2018;50:1-10.

43. Liu HL, Chen XY, Li JR, et al. Efficacy and safety of pulmonary arterial hypertension-specific therapy in pulmonary arterial hypertension: a metaanalysis of randomized controlled trials. Chest. 2016;150(2):353-66.

44. Macchia A, Marchioli R, Marfisi R, Scarano M, Levantesi G, Tavazzi L, Tognoni G. A meta-analysis of trials of pulmonary hypertension: a clinical condition looking for drugs and research methodology. Am Heart J. 2007;153(6):1037-47.

45. Macchia A, Marchioli R, Tognoni G, Scarano M, Marfisi R, Tavazzi L, Rich S. Systematic review of trials using vasodilators in pulmonary arterial hypertension: why a new approach is needed. Am Heart J. 2010;159(2):245-57.

46. Pan Y, Hu C, Chen PH, Gu YH, Qiao QY, Pan LH, Zhou DC, Gu HF, Fu SK, Jin $\mathrm{HM}$. Association of oral endothelin receptor antagonists with risks of cardiovascular events and mortality: meta-analysis of randomized controlled trials. Eur J Clin Pharmacol. 2017;73(3):267-78.

47. Pan J, Lei L, Zhao C. Comparison between the efficacy of combination therapy and monotherapy in connective tissue disease associated pulmonary arterial hypertension: a systematic review and meta-analysis. Clin Exp Rheumatol. 2018;14:14.

48. Paramothayan NS, Lasserson TJ, Wells AU, Walters EH. Prostacyclin for pulmonary hypertension in adults. Cochrane Database Syst Rev (Online). 2009:(2):CD002994.

49. Petrovic M, Locatelli I. A Bayesian network meta-analysis of add-on drug therapies specific for pulmonary arterial hypertension. Ann Pharmacother. 2020a;54(5):423-33.

50. Petrovic M, Locatelli I. Comparative effectiveness of pulmonary arterial hypertension drugs in treatment-naive patients: a network meta-analysis. J Comp Eff Res. 2020b;9(1):7-22. 
51. Ryerson CJ, Nayar S, Swiston JR, Sin DD. Pharmacotherapy in pulmonary arterial hypertension: a systematic review and meta-analysis. Respir Res. 2010;11 (no pagination):12.

52. Savarese G, Paolillo S, Costanzo P, D'Amore C, Cecere M, Losco T, Musella F, Gargiulo P, Marciano C, Perrone-Filardi P. Do changes of 6-minute walk distance predict clinical events in patients with pulmonary arterial hypertension?: a meta-analysis of 22 randomized trials. J Am Coll Cardiol. 2012;60(13):1192-201.

53. Savarese G, Musella F, C DA, Losco T, Marciano C, Gargiulo P, Rengo G, Dellegrottaglie S, Bossone E, Leosco D, et al. Haemodynamics, exercise capacity and clinical events in pulmonary arterial hypertension. Eur Respir J. 2013;42(2):414-24.

54. Silva MA, Donovan JL, Durie R, Ventura D, Alspach RM, Kanaan AO, Njoroge M. A mixed treatment comparison meta-analysis of pharmacotherapeutic monotherapy and placebo for pulmonary artery hypertension. Clin Pulm Med. 2017;24(4):149-59.

55. Steele P, Strange G, Wlodarczyk J, Dalton B, Stewart S, Gabbay E, Keogh A Hemodynamics in pulmonary arterial hypertension (PAH): do they explain long-term clinical outcomes with PAH-specific therapy? BMC CardiovasC Disord. 2010;10:9.

56. Thom HH, Capkun G, Cerulli A, Nixon RM, Howard LS. Network metaanalysis combining individual patient and aggregate data from a mixture of study designs with an application to pulmonary arterial hypertension. BMC Med Res Methodol. 2015;15:34.

57. Tran K, Coyle K, Jabr MF, Coyle D, Boucher M, Mielniczuk L, Swiston JR, Rabb D, Cimon K, Blouin J, Innes M. Drugs for pulmonary arterial hypertension: comparative efficacy, safety, and cost-effectiveness: Canadian Agency for Drugs and Technologies in Health; 2015.

58. Vizza CD, Badagliacca R, Messick CR, Rao Y, Nelsen AC, Benza RL. The impact of delayed treatment on 6-minute walk distance test in patients with pulmonary arterial hypertension: a meta-analysis. Int J Cardiol. 2018;254:299-301.

59. Wang S, Yu M, Zheng X, Dong S. A Bayesian network meta-analysis on the efficacy and safety of eighteen targeted drugs or drug combinations for pulmonary arterial hypertension. Drug Deliv. 2018;25(1):1898-909.

60. Wei A, Gu Z, Li J, Liu X, Wu X, Han Y, Pu J. Clinical adverse effects of endothelin receptor antagonists: insights from the meta-analysis of 4894 patients from 24 randomized double-blind placebo-controlled clinical trials. J Am Heart Assoc. 2016;5 (11) (no pagination):e003896.

61. Xing $X Q$, Han $B, W u X W$, Xiao $Y$, Wu SJ. Efficacy and safety of prostacyclins therapy in pulmonary arterial hypertension: a meta-analysis. Afr J Pharm Pharmacol. 2011;5(20):2199-208.

62. Zhang HD, Zhang R, Jiang X, Sun K, Wu DC, Jing ZC. Effects of oral treatments on clinical outcomes in pulmonary arterial hypertension: a systematic review and meta-analysis. Am Heart J. 2015;170(1):96-103.e114.

63. Zhang H, Li X, Huang J, Li H, Su Z, Wang J. Comparative efficacy and safety of prostacyclin analogs for pulmonary arterial hypertension: a network meta-analysis. Medicine. 2016;95(4):e2575.

64. Zhang YJ, Wang N, Gu ZC, Wei AH, Cheng AN, Fang SS, Du HL, Wang LZ, Zhang GQ. A network meta-analysis for safety of endothelin receptor antagonists in pulmonary arterial hypertension. Cardiovasc Diagn Ther. 2019;9(3):239-49.

65. Zheng $Y$, Xiong CM. Oral targeted therapies in the treatment of pulmonary arterial hypertension: a meta-analysis of clinical trials. Eur Heart J. 2014a;1: $10-1$.

66. Zheng Y, Yang T, Chen G, Hu E, Gu Q, Xiong C. Prostanoid therapy for pulmonary arterial hypertension: a meta-analysis of survival outcomes. Eur J Clin Pharmacol. 2014b;70(1):13-21.

67. Zheng YG, Ma H, Chen L, Jiang XM, Zhou L, Lin S, Chen SL. Efficacy and safety of oral targeted therapies in pulmonary arterial hypertension: a metaanalysis of randomized clinical trials. Pulm Circ. 2018;8(4):2045894018798183.

68. Zhu B, Wang L, Sun L, Cao R. Combination therapy improves exercise capacity and reduces risk of clinical worsening in patients with pulmonary arterial hypertension: a meta-analysis. J Cardiovasc Pharmacol. 2012;60(4): 342-6.

69. Liu C, Chen J, Gao Y, Deng B, Liu K. Endothelin receptor antagonists for pulmonary arterial hypertension. Cochrane Database Syst Rev. 2013;(2)

70. Singh T, Rohit M, Grover A, Malhotra S, Vijayvergiya R. A randomized, placebo-controlled, double-blind, crossover study to evaluate the efficacy of oral sildenafil therapy in severe pulmonary artery hypertension. Am Heart J. 2006;151(4):851.e851-5.
71. Galie N, Badesch D, Oudiz F, Simonneau G, McGoon M, Keogh A, Frost A, Zwicke D, Naeije R, Shapiro S, et al. Ambisentan therapy for pulmonary arterial hypertension. J Am Coll Cardiol. 2005a;46(3):529-35.

72. Pulido T, Adzerikho I, Channick R, Delcroix M, Galie N, Ghofrani H, Jansa P, Jing Z. Macitentan and morbidity and mortality in pulmonary arterial hypertension. N Engl J Med. 2013;369:809-18.

73. Galie N, Barbera J, Frost A, Ghofrani H, Hoeper M, McLaughlin A, Peacock A, Simonneau G. Initial use of Ambrisentan plus Tadalafil in pulmonary arterial hypertension. N Engl J Med. 2015a;373(9):834-44.

74. Sitbon O, Channick R, Chin KM, Frey A, Gaine S, Galiè N, Ghofrani H-A, Hoeper MM, Lang IM, Preiss $R$, et al. Selexipag for the treatment of pulmonary arterial hypertension. N Engl J Med. 2015;373(26):2522-33.

75. Cipriani A, Higgins JP, Geddes JR, Salanti G. Conceptual and technical challenges in network meta-analysis. Ann Intern Med. 2013;159(2):130-7.

76. Salanti G, Del Giovane C, Chaimani A, Caldwell DM, Higgins JP. Evaluating the quality of evidence from a network meta-analysis. PLoS One. 2014;9(7): e99682.

77. Rubin L, Mendoza J, Hood M, McGoon M, Barst R, Williams W, Diehl J, Crow J, Long W. Treatment of primary pulmonary hypertension with continuous intravenous prostacyclin (epoprostenol). Ann Intern Med. 1990;112(485):491.

78. Barst R, Rubin L, Long W, McGoon M, Rich S, Badesch D, Groves B, Tapson $V$, Bourge $R$, Brundage B. A comparison of continuous intravenous epoprostenol (prostacyclin) with conventional therapy for primary pulmonary hypertension. N Engl J Med. 1996;334(5):296-301.

79. Anderson RJ, Malhotra A, Kim NH. Pulmonary hypertension: evolution of pulmonary arterial hypertension and chronic thromboembolic pulmonary hypertension. J Thorac Dis. 2016;8(Suppl 7):S562-5.

80. Humbert M, Barst R, Robbins I, Channick R, Galie N, Boonstra A, Rubin L, Horn E, Manes A, Simonneau G. Combination of bosentan with epoprostenol in pulmonary arterial hypertension: BREATHE-2. Eur Respir J. 2004;24:353-9.

81. Simonneau G, Rubin L, Galie N, Barst R, Fleming T, Frost A, Engel P, Kramer $M$, Burgess $G$, Collings $L$, et al. Addition of sildenafil to long-term intravenous epoprostenol therapy in patients with pulmonary arterial hypertension. Ann Intern Med. 2008;149(8):521-30.

82. McLaughlin V, Channick R, Ghofrani H, Lemarie J, Naeije R, Packer M, Souza R, Tapson V, Tolson J, Al Hiti H, et al. Bosentan added to sildenafil therapy in patients with pulmonary arterial hypertension. Eur Respir J. 2015; In Press.

83. LeVarge BL, Channick RN. The changing paradigm in pulmonary hypertension trials: longer duration, new endpoints. Curr Opin Pulm Med. 2015;21(5):438-45.

84. McLaughlin W, Badesch DB, Delcroix M, Fleming TR, Gaine SP, Galie N, Gibbs JS, Kim NH, Oudiz RJ, Peacock A, et al. End points and clinical trial design in pulmonary arterial hypertension. J Am Coll Cardiol. 2009;54(1 Suppl):S97-107.

85. EMA: Guideline on the clinical investigations of medicinal products for the treatment of pulmonary arterial hypertension. 2009. Available from: https:// www.ema.europa.eu/documents/scientific-guideline/guideline-clinicalinvestigations-medicinal-products-treatment-pulmonary-arterialhypertension_en.pdf.

86. Sitbon O, Gomberg-Maitland M, Granton J, et al. Clinical trial design and new therapies for pulmonary arterial hypertension. Eur Respir J. 2019;53(1): 1801908.

87. Fritz JS, Blair C, Oudiz RJ, Dufton C, Olschewski H, Despain D, Gillies H, Kawut SM. Baseline and follow-up 6-min walk distance and brain natriuretic peptide predict 2-year mortality in pulmonary arterial hypertension. Chest. 2013;143(2):315-23.

88. Olschewski H, Simonneau G, Galie N, Higenbottam T, Naeije R, Rubin L, Nikkho S, Speich R, Hoeper M. Inhaled Iloprost for severe pulmonary hypertension. N Engl J Med. 2002;347(5):322-9.

\section{Publisher's Note}

Springer Nature remains neutral with regard to jurisdictional claims in published maps and institutional affiliations. 